\title{
A systematic review of the role of videolaryngoscopy in successful orotracheal intubation
}

\author{
David W Healy ${ }^{*}$, Oana Maties, David Hovord and Sachin Kheterpal
}

\begin{abstract}
Background: The purpose of our study was to organize the literature regarding the efficacy of modern videolaryngoscopes in oral endotracheal intubation, then perform a quality assessment according to recommended external criteria and make recommendations for use.

Methods: Inclusion criteria included devices with recent studies of human subjects. A total of 980 articles were returned in the initial search and 65 additional items were identified using cited references. After exclusion of articles failing to meet study criteria, 77 articles remained. Data were extracted according to the rate of successful intubation and improvement of glottic view compared with direct laryngoscopy. Studies were classified according to whether they primarily examined subjects with normal airways, possessing risk factors for difficult direct laryngoscopy, or following difficult or failed direct laryngoscopy.

Results: The evidence of efficacy for videolaryngoscopy in the difficult airway is limited. What evidence exists is both randomized prospective and observational in nature, requiring a scheme that evaluates both forms and allows recommendations to be made.

Conclusions: In patients at higher risk of difficult laryngoscopy we recommend the use of the Airtraq, CTrach, GlideScope, Pentax AWS and V-MAC to achieve successful intubation. In difficult direct laryngoscopy $(C \& L>/=3)$ we cautiously recommend the use of the Airtraq, Bonfils, Bullard, CTrach, GlideScope, and Pentax AWS, by an operator with reasonable prior experience, to achieve successful intubation when used in accordance with the ASA practice guidelines for management of the difficult airway. There is additional evidence to support the use of the Airtraq, Bonfils, CTrach, GlideScope, McGrath, and Pentax AWS following failed intubation via direct laryngoscopy to achieve successful intubation. Future investigation would benefit from precise qualification of the subjects under study, and an improvement in overall methodology to include randomization and blinding.
\end{abstract}

Keywords: Laryngoscopy, Airway management, Intubation, Technology

\section{Background}

Since Macintosh [1] (1943) and Miller [2] (1941) envisioned and developed their direct laryngoscopes attempts have been made to improve on these techniques and equipment using technological advances. Nevertheless, these original techniques have withstood the test of time and remain the mainstay of intubation globally. Direct laryngoscopy (DL) relies on the formation of a "lineof-sight" between the operator and the laryngeal inlet,

\footnotetext{
*Correspondence: dhealy@med.umich.edu

Department of Anesthesiology, University of Michigan Hospital, 1500 East Medical Center Drive 1H247, Box 0048, Ann Arbor, Michigan 48109, USA
}

success reliant on careful head positioning and consistent anatomy. When these conditions are not met, for example in poor tissue mobility, limited mouth opening, or enlarged tongue, the failure rate of intubation with conventional direct laryngoscopy increases [3-5].

Videolaryngoscopy (VL) is a relatively recent development that attempts to improve the success of tracheal intubation. High-resolution micro cameras and small portable flat-screen monitors are used in an attempt to improve upon the view and success rate of direct laryngoscopy. Similar technologies have been successfully applied to other fields of medicine such as laparoscopic 
and robotic surgery, making new techniques and procedures possible [6]. The use of Videolaryngoscopy produces a view of the laryngeal inlet independent of the line of sight, particularly when an angulated device is used. This may free them from some of the conditions essential to the success of direct laryngoscopy. There is an assumption that improved lighting and a better view can improve the success of laryngoscopy. This may be incorrect as an improvement in success may be limited by both use of unfamiliar equipment and difficulty placing an endotracheal tube out of the line of sight. Some previous reviews have indicated an advantage when using videolaryngoscopy [7-9] but a need remains for an systematic evidence based review of the efficacy of videolaryngoscopy above that of direct laryngoscopy.

To appreciate any benefit from the use of videolaryngoscopy we need to appreciate the mechanism and incidence of failure of direct laryngoscopy. The incidence of difficulty encountered during direct laryngoscopy is difficult to ascertain as it depends upon both definition and patient selection. The best evidence available is from a meta-analysis of 50,760 patients in which difficulty at laryngoscopy occurred in $5.8 \%$ (95\% CI 4.5 - 7.5) of subjects [10]. Of note, the metanalysis did exclude all patients whose airways were "anatomically abnormal" or in whom DL was thought inappropriate. The definition of difficult laryngoscopy was broad and included all subjects with Cormack and Lehane views 3 or greater. The actual incidence of difficult intubation in this difficult laryngoscopy group is presumably less than $5.8 \%$ as many patients with a Cormack and Lehane view 3 can successfully be intubated with direct laryngoscopy and the use of a gum elastic bougie by a reasonably experience practitioner. However, even given this broad definition of difficult laryngoscopy, this still suggests an impressive overall intubation success rate of $>95 \%$ [11] for direct laryngoscopy among patients thought to be suitable for this technique. It is this high standard against which the new methods of videolaryngoscopy must be assessed.

\section{Methods}

Pubmed and Cochrane review searches were made of all published articles regarding Video Laryngoscopy (VL) from 1999 to April 2011. The following search terms were used: Airtraq, Berci DCI, Bonfils Fibre(er) scope, Bonfils Intubation, Bullard laryngoscope, C-MAC, C-MAC D-blade, CTrach, video laryngoscopy, EVO videolaryngoscope, GlideScope, Glidescope Direct, LMA CTrach, McGrath laryngoscope, McGrath MAC, McGrath series 5, Pentax Airway Scope, Pentax AWS, Rusch, Shikani, Storz Berci, Storz CMAC, Styletscope, V-MAC, Upsherscope, WuScope. $\mathrm{X}$-Lite. Additional search items, for classification purposes, were cervical, cervical limitation, cervical stabilization, obesity, difficult intubation, failed intubation, failed ventilation and education. From this selection all articles were reviewed including randomized controlled trials, observational studies, review articles, meta-analyses and editorials. Bibliographies were checked manually for any relevant articles. Articles published in the ASA meeting proceedings were included and a search made of all ongoing clinical trials in Clinicaltrials.gov.

\section{Inclusion criteria}

Orotracheal intubation.

Procedure performed by trained operators.

English language or accessible translation of key outcomes and methodology.

Device had at least 10 articles published on its use during the previous 5 years (until April 2011).

\section{Exclusion criteria}

Studies of patients aged less than 18 years.

Duplicates, unrelated studies, abstracts, single case reports and small studies (less than 5 subjects).

Manikin studies.

\section{Data extraction}

To summarize the data available from the multiple studies the following measures were extracted from each article:

An overall measure of study quality (based on SIGN recommendations [12]).

Study device.

Summary of method.

Number of subjects in study group.

Number of predicted normal airway (eg MP 1/2).

Number of predicted difficult (eg MP 3/4).

Number of difficult direct laryngoscopies (known or C\&L $>3$ on Mac DL).

Improvement in laryngeal view compared with direct laryngoscopy.

Average time to intubation. Expressed as central tendency (Mean or median) and variability (95\% confidence interval or Inter Quartile Range).

Success rate (percentage) on 1st attempt and overall success (OA).

\section{Results}

A total of 980 articles were returned in the initial Pub Med search and 65 additional items were identified using references cited in these articles. After exclusion of articles failing to meet study criteria, 77 articles remained (Figure 1, Tables 1, 2 and 3).

\section{Discussion}

\section{The choice of devices to study}

When considering the wide variety of airway devices currently available, it is impossible to perform a systematic 


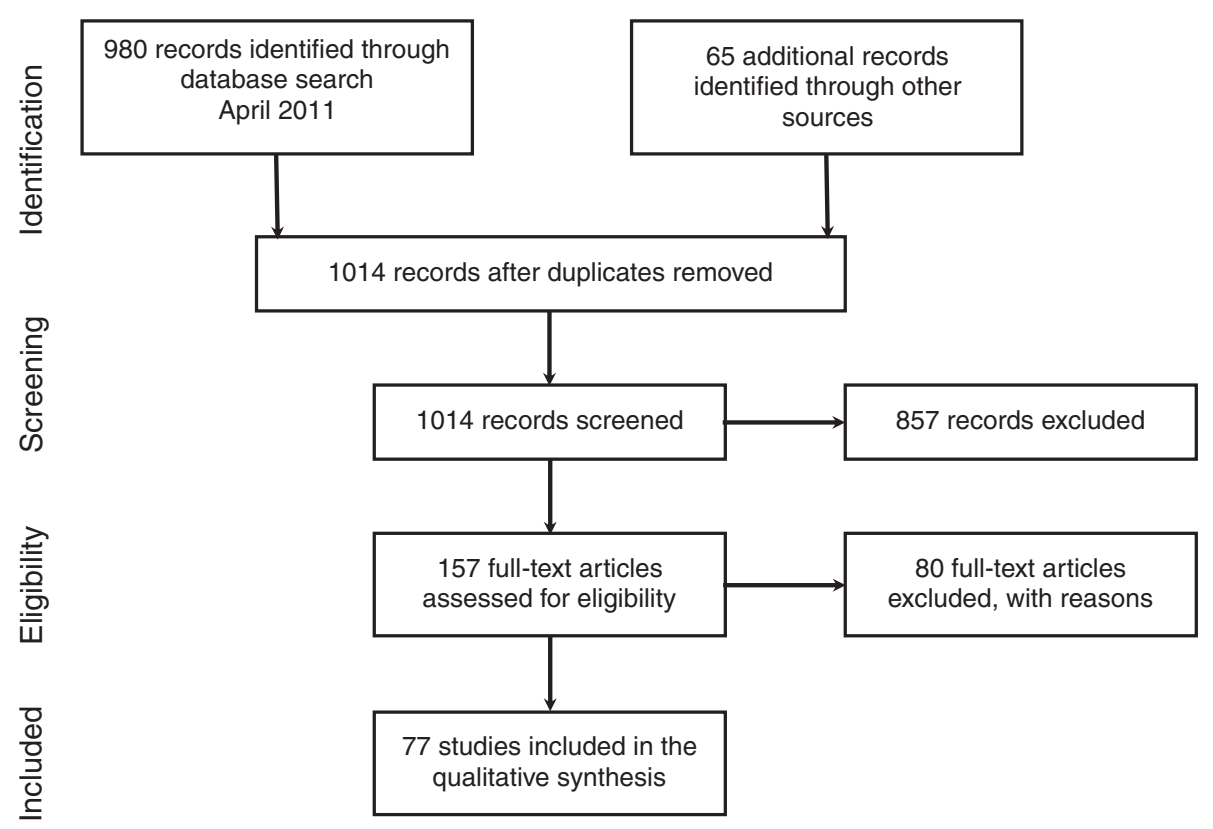

Figure 1 Information flow through the systematic review.

review of all. With this is mind we limited our review to videolaryngoscopy and applied a rigid, objective inclusion criterion (that of at least 10 publications in the last 5 years) in an attempt to make the selection contemporary. No performance assessment of such a diverse group of devices will ever be perfect, but we have attempted to limit through our objective inclusion criteria those devices that have received the most recent development and where competition exists between different versions of similar equipment. The dynamic nature of the field is illustrated by the decision to discontinue the manufacture of the CTrach by LMA North America in December 2009 during the period of the review. However, the CTrach is still in clinical use and fulfilled the study inclusion criteria so remained in this systematic review. The main technique excluded from this review is flexible fiberoptic bronchoscopy. We feel this method has a specific clinical application and is difficult to compare with standard direct laryngoscopy. The inclusion criteria limited the choice of devices to the GlideScope, V-MAC (including C-MAC and Storz Berci DCI), Bullard, McGrath, Bonfils, Airtraq, Pentax AWS, LMA CTRACH. The recently introduced CMAC and the older Storz DCI and V-MAC were considered versions of the Storz Macintosh video laryngoscope for the purpose of this review and referred to as the V-MAC (video Macintosh) for the remainder of this review.

The devices returned by our methodology are presented in the following diagram (Figure 2). We have classified the videolaryngoscopes according their principle shape and form:
1. Presence of an integrated channel (to guide placement of the endotracheal tube).

2. The form of a videostylet (with the endotracheal tube placed around the device).

3. A rigid blade laryngoscopes (without a channel, the endotracheal tube requiring some kind of independent stylet to guide placement).

Rigid blade laryngoscopes are sub-divided into those with a "standard" blade and those with an angled blade as classified by Niforopoulou and colleagues [8]. There may be differences between the two types with respect to the glottic view on laryngoscopy and ease of intubation. Overall, we feel this classification scheme adds some clarity to the set of devices under examination. Clearly there are design differences between each device within these broad groups (for example presence of antifogging device etc.) Our classification is not novel as previous classifications of videolaryngoscopy have already been published $[9,11]$ but use different criteria to differentiate.

\section{Subject classification Predicting difficult direct laryngoscopy}

This low incidence of difficulty encountered at direct laryngoscopy makes the study of true difficulty problematic. Our standard airway examination is a poor predictor of its occurrence [11,97]. The data from a meta-analysis of 50,760 patients found that the positive predictive value (PPV) of the Mallampati score (at predicting a Cormack and Lehane view 3 or 
Table 1 Data extraction

\begin{tabular}{|c|c|c|c|c|c|c|c|c|c|c|}
\hline 1st Author & Quality & Device & Method & $\begin{array}{l}\mathrm{N} \\
\text { device }\end{array}$ & $\begin{array}{l}\text { Predicted } \\
\text { easy (MP 1-2) }\end{array}$ & $\begin{array}{l}\text { Predicted } \\
\text { difficult } \\
\text { (MP 3-4) }\end{array}$ & $\begin{array}{l}\text { Difficult } \\
\text { laryngoscopy } \\
\text { (C\&L 3-4 on DL) }\end{array}$ & $\begin{array}{l}\text { Achievement of } \\
\text { C\&L I view }\end{array}$ & $\begin{array}{l}\text { Time to intubation } \\
95 \% \mathrm{Cl} \text { or IQR }\end{array}$ & $\begin{array}{l}\text { Success\% } 1 \text { st attempt } \\
\text { Overall (OA) }\end{array}$ \\
\hline \multirow[t]{2}{*}{ Maharaj (2006) [13] } & \multirow[t]{2}{*}{$(+)$} & \multirow[t]{2}{*}{ Airtraq } & \multirow{2}{*}{$\begin{array}{l}\text { Randomized, } 60 \\
\text { subjects, Airtraq v } \\
\text { Mac DL }\end{array}$} & \multirow[t]{2}{*}{30} & \multirow[t]{2}{*}{30} & \multirow[t]{2}{*}{0} & \multirow[t]{2}{*}{ No data } & $95 \%$ & Mean 12.2 & $100 \% 1 s t$ \\
\hline & & & & & & & & Mac DL 70\% & (95\% Cl 9.1 to 15.3 ) & $100 \% \mathrm{OA}$ \\
\hline \multirow[t]{2}{*}{ Maharaj (2007) [14] } & \multirow[t]{2}{*}{$(+)$} & \multirow[t]{2}{*}{ Airtraq } & \multirow{2}{*}{$\begin{array}{l}\text { Randomized, } 40 \\
\text { subjects, Airtraq } \vee \\
\text { Mac DL, Cervical spine } \\
\text { limitation (MILS) }\end{array}$} & \multirow[t]{2}{*}{20} & \multirow[t]{2}{*}{20} & \multirow[t]{2}{*}{0} & \multirow[t]{2}{*}{ No data } & $95 \%$ & Mean 13.2 & \multirow[t]{2}{*}{ No data } \\
\hline & & & & & & & & Mac DL 30\% & (95\% Cl 10.6 to 15.7$)$ & \\
\hline \multirow[t]{2}{*}{ Maharaj (2007) [15] } & \multirow[t]{2}{*}{ na } & \multirow[t]{2}{*}{ Airtraq } & \multirow{2}{*}{$\begin{array}{l}\text { Observational, Case } \\
\text { series, } 7 \text { subjects, } \\
\text { failed Mac DL }\end{array}$} & \multirow[t]{2}{*}{7} & \multirow[t]{2}{*}{0} & \multirow[t]{2}{*}{7} & \multirow[t]{2}{*}{4 C\&L 4} & $100 \%$ & Mean 14 & \multirow[t]{2}{*}{ No data } \\
\hline & & & & & & & & Mac DL 0\% & (95\% Cl 8.5 to 18.9$)$ & \\
\hline \multirow[t]{2}{*}{ Ndoko (2007) [16] } & \multirow[t]{2}{*}{$(-)$} & \multirow[t]{2}{*}{ Airtraq } & \multirow{2}{*}{$\begin{array}{l}\text { Randomized, } 70 \\
\text { subjects, Mac DL v } \\
\text { Airtraq, risk of } \\
\text { difficulty }\end{array}$} & \multirow[t]{2}{*}{35} & 0 & 35 & No data & No data & Mean 30 & $100 \% \mathrm{OA}$ \\
\hline & & & & & & & & & (95\% Cl 21.4 to 35.8 ) & \\
\hline Arslan (2009) [17] & $(+)$ & Airtraq & Randomized, 86 & 43 & 42 & 1 & No data & No data & Mean 25.6 & No data \\
\hline & & & $\begin{array}{l}\text { subjects, Alrtraq v } \\
\text { CTrach, Cervical spine } \\
\text { limitation (collar) }\end{array}$ & & & & & & (95\% Cl 21.4 to 29.8) & \\
\hline Dhonneur (2009) [18] & $(+)$ & Airtraq & Randomized, 318 & 106 & 82 & 24 & No data & $94 \%$ & Mean 29 & No data \\
\hline & & & $\begin{array}{l}\text { subjects, Alltraq v } \\
\text { Mac DL v CTrach, } \\
\text { obese }\end{array}$ & & & & & Mac DL 51\% & (95\% Cl 26.7 to 31.3) & \\
\hline Lange (2009) [19] & $(+)$ & Airtraq & Randomized, 60 & 30 & 26 & 4 & 4 C\&L 3-4 & $90 \%$ & Mean 19.7 & No data \\
\hline & & & Airtraq $\vee$ GlideScope & & & & & Mac DL 57\% & (95\% Cl to 15.7 to 23.8 ) & \\
\hline Malin (2009) [20] & na & Airtraq & Observational, Case & 47 & 0 & 47 & 47 C\&L 2b-4 & $85 \%$ & No data & $95 \% 1$ st \\
\hline & & & failed Mac DL & & & & & Mac DL 0\% & & $100 \% \mathrm{OA}$ \\
\hline Turkstra (2009) [21] & $(+)$ & Airtraq & Randomized, cross- & 24 & 24 & 0 & 2 & $90 \%$ & Median 8.8 & $100 \% 1 s t$ \\
\hline & & & $\begin{array}{l}\text { Airtraq } \vee \text { Mac, cervical } \\
\text { spine limitation (MILS) }\end{array}$ & & & & & Mac DL 20\% & (IQR 6.7 to 10.6) & $100 \% \mathrm{OA}$ \\
\hline Chalkeidis (2010) [22] & $(+)$ & Airtraq & Randomized, 63 & 35 & 25 & 10 & No data & No data & Mean 30 & $80 \%$ OA \\
\hline & & & $\begin{array}{l}\text { subjects, Airtraq v } \\
\text { Mac DL }\end{array}$ & & & & & & (95\% Cl 27.1 to 32.9$)$ & \\
\hline Koh (2010) [23] & $(+)$ & Airtraq & Randomized, 50 & 25 & 20 & 5 & No data & No data & Mean 50 & $100 \% \mathrm{OA}$ \\
\hline & & & $\begin{array}{l}\text { subjects, Airtraq v } \\
\text { Mac DL, Cervical spine } \\
\text { limitation (collar) }\end{array}$ & & & & & & ( $95 \% \mathrm{Cl}$ to 36.2 to 63.8 ) & \\
\hline Halligan (2003) [24] & na & Bonfils & Observational, Case & 60 & 58 & 2 & No data & No data & Median 33 & $98 \% \mathrm{OA}$ \\
\hline & & & & & & & & & (IQR 24 to 50) & \\
\hline
\end{tabular}


Table 1 Data extraction (Continued)

\begin{tabular}{|c|c|c|c|c|c|c|c|c|c|c|}
\hline Wong (2003) [25] & na & Bonfils & $\begin{array}{l}\text { Observational, Case } \\
\text { series, } 36 \text { subjects }\end{array}$ & 36 & No data & No data & No data & No data & $\begin{array}{l}\text { Median } 80 \\
\text { (No IQR report) }\end{array}$ & $86 \% \mathrm{OA}$ \\
\hline \multirow[t]{2}{*}{ Bein (2004) [26] } & \multirow[t]{2}{*}{$(-)$} & \multirow[t]{2}{*}{ Bonfils } & \multirow{2}{*}{$\begin{array}{l}\text { Randomized, } 80 \\
\text { subjects, Bonfils v } \\
\text { ILMA, Risk of difficulty }\end{array}$} & \multirow[t]{2}{*}{40} & \multirow[t]{2}{*}{12} & \multirow[t]{2}{*}{28} & \multirow[t]{2}{*}{ No data } & \multirow[t]{2}{*}{ No data } & Median 40 & $98 \% 1 \mathrm{st}$ \\
\hline & & & & & & & & & (IQR 23 to 77) & $100 \% \mathrm{OA}$ \\
\hline \multirow[t]{2}{*}{ Bein (2004) [27] } & \multirow[t]{2}{*}{ na } & \multirow[t]{2}{*}{ Bonfils } & \multirow{2}{*}{$\begin{array}{l}\text { Observational, Case } \\
\text { series, } 25 \text { subjects, } \\
\text { failed Mac DL }\end{array}$} & \multirow[t]{2}{*}{25} & \multirow[t]{2}{*}{0} & \multirow[t]{2}{*}{ No data } & \multirow[t]{2}{*}{25} & \multirow[t]{2}{*}{ No data } & Median 48 & \multirow[t]{2}{*}{ No data } \\
\hline & & & & & & & & & (IQR 30 to 80) & \\
\hline \multirow[t]{2}{*}{ Wahlen (2004) [28] } & $(-)$ & Bonfils & Randomized, 48 & 12 & 12 & 0 & No data & No data & Mean 52 & $92 \% \mathrm{OA}$ \\
\hline & & & $\begin{array}{l}\text { subjects, Bonflls v Mlac } \\
\text { DL v Bullard v ILMA }\end{array}$ & & & & & & (95\% Cl 38.1 to 66.1$)$ & \\
\hline Byhahn (2008) [29] & $(+)$ & Bonfils & Randomized, 76 & 38 & 38 & 0 & Mac group 17 & $82 \%$ & Mean 64 & $71 \% 1 s t$ \\
\hline & & & $\begin{array}{l}\text { subjects, Bontlls v Mlac } \\
\text { DL, Cervical spine } \\
\text { limitation (collar) }\end{array}$ & & & & & Mac DL 5\% & (95\% Cl 56.1 to 71.9 ) & $82 \% \mathrm{OA}$ \\
\hline Corbanese (2009) [30] & na & Bonfils & Observational, Case & 100 & 100 & 0 & No data & No data & Median 30 & $89 \% 1$ st \\
\hline & & & & & & & & & (IQR 25 to 40) & $98 \%$ OA \\
\hline Corso (2010) [31] & na & Bonfils & $\begin{array}{l}\text { Observational, Case } \\
\text { series, } 10 \text { subjects }\end{array}$ & 10 & No data & No data & No data & No data & No data & No data \\
\hline MacQuarrie (1999) & na & Bullard & Observational, Case & $40 \times 2$ & 28 & No data & 52 & No data & MFIS group & $89 \% 1 s t$ \\
\hline & & & $\begin{array}{l}\text { serıes, } 80 \text { subjects, } \\
\text { Cervical spine }\end{array}$ & grps & & & & & Mean 41 & $98 \% \mathrm{OA}$ \\
\hline & & & limitation (collar) & & & & & & (95\% Cl 35.3 to 46.7 ) & \\
\hline & & & & & & & & & ISETT group & \\
\hline & & & & & & & & & $\begin{array}{l}\text { Mean } 45.4(95 \% \text { Cl } 39.4 \text { to } \\
\text { 51.4) }\end{array}$ & \\
\hline Shulman (2001) [33] & $(-)$ & Bullard & Randomized, cross- & $25 \times 2$ & No data & No data & No data & No data & Standard Group: Mean 37 & $85 \% \mathrm{OA}$ \\
\hline & & & $\begin{array}{l}\text { Over, } 50 \text { subjects, } \\
\text { Bullard v FOl, Cervical }\end{array}$ & grps & & & & & (95\% Cl 26.2 to 47.8$)$ & \\
\hline & & & spine limitation (MILS) & & & & & & Cricoid Group & \\
\hline & & & & & & & & & $\begin{array}{l}\text { Mean } 38 \text { (95\% Cl } 26.9 \text { to } \\
\text { 49.1) }\end{array}$ & \\
\hline Wahlen (2004) [28] & $(+)$ & Bullard & Randomized, 48 & 12 & 12 & 0 & No data & $92 \%$ & Mean 16.1 & $92 \% 1 s t$ \\
\hline & & & $\begin{array}{l}\text { subjects, Bullard v } \\
\text { Mac v Bonfils v ILMA }\end{array}$ & & & & & Mac DL 33\% & (95\% Cl 12.1 to 20$)$ & $92 \% \mathrm{OA}$ \\
\hline Nileshwar (2007) [34] & $(+)$ & Bullard & Randomized, 62 & 31 & 19 & No data & 12 & No data & Mean 84 & $86 \% 1$ st \\
\hline & & & $\begin{array}{l}\text { Bullard } v \text { ILMA, } \\
\text { cervical spine } \\
\text { limitation (MILS) }\end{array}$ & & & & & & (95\% Cl 66.4 to 101.6) & $90 \%$ OA \\
\hline Teoh (2010) [35] & $(+)$ & C-MAC & Randomized, 400 & 100 & 85 & 15 & No data & $87 \%$ & Mean 31.9 & $93 \% 1$ st \\
\hline & & & & & & & & & (95\% Cl 28.4 to 35.4 ) & $100 \% \mathrm{OA}$ \\
\hline
\end{tabular}


Table 1 Data extraction (Continued)

\begin{tabular}{|c|c|c|c|c|c|c|c|c|c|c|}
\hline & & & $\begin{array}{l}\text { Pentax AWS } \vee \text { C-MAC } \\
\vee \text { MacDL }\end{array}$ & & & & & & & \\
\hline \multirow[t]{2}{*}{ Dhonneur (2006) [36] } & \multirow[t]{2}{*}{$(+)$} & \multirow[t]{2}{*}{ CTrach } & \multirow{2}{*}{$\begin{array}{l}\text { Randomized, } 104 \\
\text { subjects, Mac DL v } \\
\text { CTrach, obese }\end{array}$} & \multirow[t]{2}{*}{52} & \multirow[t]{2}{*}{43} & \multirow[t]{2}{*}{9} & \multirow[t]{2}{*}{ No data } & \multirow[t]{2}{*}{$75 \%$} & Mean 176 & \multirow[t]{2}{*}{ No data } \\
\hline & & & & & & & & & (95\% Cl 166 to 186$)$ & \\
\hline Goldman (2006) [37] & na & CTrach & $\begin{array}{l}\text { Observational, Case } \\
\text { series, } 328 \text { subjects }\end{array}$ & 328 & No data & No data & No data & $91 \%$ & No data & No data \\
\hline \multirow[t]{2}{*}{ Goldman (2006) [38] } & \multirow[t]{2}{*}{ na } & \multirow[t]{2}{*}{ CTrach } & \multirow{2}{*}{$\begin{array}{l}\text { Observational, Case } \\
\text { series, } 6 \text { subjects }\end{array}$} & \multirow[t]{2}{*}{6} & \multirow[t]{2}{*}{3} & \multirow[t]{2}{*}{3} & \multirow[t]{2}{*}{6} & $100 \%$ & \multirow[t]{2}{*}{ No data } & \multirow[t]{2}{*}{ No data } \\
\hline & & & & & & & & Mac DL 0\% & & \\
\hline \multirow[t]{2}{*}{ Liu (2006) [39] } & \multirow[t]{2}{*}{ na } & \multirow[t]{2}{*}{ CTrach } & \multirow{2}{*}{$\begin{array}{l}\text { Observational, Case } \\
\text { series, } 100 \text { subjects }\end{array}$} & \multirow[t]{2}{*}{100} & \multirow[t]{2}{*}{84} & \multirow[t]{2}{*}{26} & \multirow[t]{2}{*}{9} & $28 \%$ & Median 166 & \multirow[t]{2}{*}{ No data } \\
\hline & & & & & & & & Mac DL 59\% & (IQR 114 to 233) & \\
\hline $\begin{array}{l}\text { Timmerman (2006) } \\
\text { [40] }\end{array}$ & na & CTrach & $\begin{array}{l}\text { Observational, Case } \\
\text { series, } 10 \text { subjects }\end{array}$ & 10 & No data & No data & No data & $30 \%$ & No data & No data \\
\hline $\begin{array}{l}\text { Timmerman (2006) } \\
\text { [41] }\end{array}$ & na & CTrach & $\begin{array}{l}\text { Observational, Case } \\
\text { series, } 60 \text { subjects }\end{array}$ & 60 & No data & No data & 3 & $55 \%$ & No data & No data \\
\hline Cattano (2007) [42] & na & CTrach & $\begin{array}{l}\text { Observational, Case } \\
\text { series, } 15 \text { subjects, } \\
\text { obese }\end{array}$ & 15 & No data & No data & No data & $60 \%$ & No data & No data \\
\hline \multirow[t]{2}{*}{ Dhonneur (2007) [43] } & \multirow[t]{2}{*}{$(+)$} & CTrach & Randomized, 120 & 60 & No data & No data & No data & $93 \%$ & Mean 119 & No data \\
\hline & & & $\begin{array}{l}\text { subjects, CIrach v } \\
\text { MacDL }\end{array}$ & & & & & & (95\% Cl 107.6 to 130.4$)$ & \\
\hline $\mathrm{Ng}(2007)$ [44] & $(-)$ & CTrach & Randomized trial, 106 & 54 & 54 & 0 & No data & $85 \%$ & Mean 73 & No data \\
\hline & & & $\begin{array}{l}\text { subjects, CIrach v } \\
\text { GlideScope }\end{array}$ & & & & & & (95\% Cl 63.2 to 82.8$)$ & \\
\hline Liu (2008) [45] & $(+)$ & CTrach & Randomized, 271 & 134 & 118 & 16 & 13 & $93 \%$ & Median 116 & $93 \% 1 s t$ \\
\hline & & & $\begin{array}{l}\text { subjects, Clrach v } \\
\text { ILMA (Fastrach) }\end{array}$ & & & & & Mac DL 59\% & (IQR 82 to 156) & $100 \% \mathrm{OA}$ \\
\hline Nickel (2008) [46] & na & CTrach & $\begin{array}{l}\text { Observational, Case } \\
\text { series, } 16 \text { subjects }\end{array}$ & 16 & No data & No data & No data & $44 \%$ & No data & No data \\
\hline Arslan (2009) [17] & $(+)$ & CTrach & Randomized, 86 & 43 & 42 & 1 & No data & No data & Mean 66.3 & $93 \% 1 s t$ \\
\hline & & & $\begin{array}{l}\text { subjects, Airtraq v } \\
\text { CTrach, Cervical spine } \\
\text { limitation (collar) }\end{array}$ & & & & & & (95\% Cl 57.3 to 75.3$)$ & $100 \% \mathrm{OA}$ \\
\hline Dhonneur (2009) [18] & $(+)$ & CTrach & Randomized, 318 & 106 & 78 & 28 & No data & $97 \%$ & Mean 109 & $100 \% \mathrm{OA}$ \\
\hline & & & $\begin{array}{l}\text { subjects, Alrtraq v } \\
\text { Mac DL v CTrach, } \\
\text { obese }\end{array}$ & & & & & Mac DL 51\%, & (95\% Cl 103.9 to 114.1$)$ & \\
\hline Liu (2009) [47] & na & CTrach & Observational, Case & 48 & 18 & 30 & 26 & in $96 \%$ & No data & No data \\
\hline & & & & & & & & Mac DL 0\% & & \\
\hline Malik (2009) [48] & $(+)$ & CTrach & Randomized, 90 & 30 & 30 & 0 & No data & $67 \%$ & Median 46 & $84 \% 1 s t$ \\
\hline & & & $\begin{array}{l}\text { subjects, Pentax AWS } \\
\text { v Mac DL v CTrach, }\end{array}$ & & & & & Mac DL 20\% & (IQR 38 to 107) & $90 \%$ OA \\
\hline
\end{tabular}


Table 1 Data extraction (Continued)

\begin{tabular}{|c|c|c|c|c|c|c|c|c|c|c|}
\hline & & & $\begin{array}{l}\text { cervical spine } \\
\text { limitation (MILS) }\end{array}$ & & & & & & & \\
\hline \multirow[t]{2}{*}{$\mathrm{Ng}$ (2009) [49] } & \multirow[t]{2}{*}{ na } & \multirow[t]{2}{*}{ CTrach } & \multirow{2}{*}{$\begin{array}{l}\text { Observational, Case } \\
\text { series, } 50 \text { subjects, } \\
\text { cervical spine } \\
\text { limitation (MILS) }\end{array}$} & \multirow[t]{2}{*}{50} & \multirow[t]{2}{*}{45} & \multirow[t]{2}{*}{5} & \multirow[t]{2}{*}{11} & $98 \%$ & \multirow[t]{2}{*}{ No data } & \multirow[t]{2}{*}{ No data } \\
\hline & & & & & & & & Mac DL 44\% & & \\
\hline \multirow[t]{2}{*}{ Swadia (2009) [50] } & \multirow[t]{2}{*}{ na } & \multirow[t]{2}{*}{ CTrach } & \multirow{2}{*}{$\begin{array}{l}\text { Observational, Case } \\
\text { series, } 20 \text { subjects }\end{array}$} & \multirow[t]{2}{*}{20} & \multirow[t]{2}{*}{20} & \multirow[t]{2}{*}{0} & \multirow[t]{2}{*}{ No data } & \multirow[t]{2}{*}{$60 \%$} & Mean 347.8 & \multirow[t]{2}{*}{ No data } \\
\hline & & & & & & & & & (95\% Cl 342.8 to 352.8$)$ & \\
\hline \multirow[t]{2}{*}{ Agro (2003) [4] } & \multirow[t]{2}{*}{ na } & \multirow[t]{2}{*}{ GlideScope } & \multirow{2}{*}{$\begin{array}{l}\text { Observational, Case } \\
\text { series, } 15 \text { subjects, C } \\
\text { spine limitation } \\
\text { (collar) }\end{array}$} & \multirow[t]{2}{*}{15} & \multirow[t]{2}{*}{ No data } & \multirow[t]{2}{*}{ No data } & \multirow[t]{2}{*}{10} & $33 \%$ & Mean 38 & \multirow[t]{2}{*}{ No data } \\
\hline & & & & & & & & Mac DL 0\% & (no SD report) & \\
\hline \multirow[t]{2}{*}{ Cooper (2005) [51] } & \multirow[t]{2}{*}{ na } & GlideScope & Observational, Case & 728 & 579 & 148 & $34 / 133$ & $86 \%$ & No data & $96 \%$ OA \\
\hline & & & & & & & & Mac DL 49\% & & \\
\hline Doyle (2005) [52] & na & GlideScope & $\begin{array}{l}\text { Observational, Case } \\
\text { series, } 747 \text { subjects }\end{array}$ & 747 & No data & No data & No data & No data & No data & $100 \% \mathrm{OA}$ \\
\hline Hsiao (2005) [53] & na & GlideScope & Observational, Case & 103 & No data & No data & 22 & $80 \%$ & No data & No data \\
\hline & & & $\begin{array}{l}\text { Mac DL then } \\
\text { GlideScope }\end{array}$ & & & & & Mac DL 52\% & & \\
\hline $\operatorname{Lim}(2005)$ [54] & $(+)$ & GlideScope & Randomized, 60 & 30 & 30 & 0 & 8 in Mac DL & $67 \%$ & Mean 41.8 & $86 \% 1$ st \\
\hline & & & $\begin{array}{l}\text { Mac DL, Cervical spine } \\
\text { limitation (MILS) }\end{array}$ & & & & group & Mac DL 13\% & (95\% Cl 34.2 to 49.4 ) & $94 \%$ OA \\
\hline Rai (2005) [55] & na & GlideScope & Observational, Case & 50 & No data & No data & 1 & $88 \%$ & Median 40 & No data \\
\hline & & & serles, 50 subjects & & & & & Mac DL 44\% & (IQR 30 to 55) & \\
\hline Sun (2005) [56] & $(+)$ & GlideScope & Randomized, 200 & 100 & 88 & 12 & 15 & $75 \%$ & Mean 46 & $94 \% 1$ st \\
\hline & & & Mac $\vee$ GlideScope & & & & & Mac DL 59\% & (95\% Cl 42 to 49$)$ & $99 \%$ OA \\
\hline Turkstra (2005) [57] & $(+)$ & GlideScope & Randomized, cross- & 18 & 16 & 2 & No data & No data & Mean 27 & No data \\
\hline & & & $\begin{array}{l}\text { DL and GlideScope, } \\
\text { cervical spine } \\
\text { limitation (MILS) }\end{array}$ & & & & & & (95\% Cl 21.0 to 33.0$)$ & \\
\hline $\mathrm{Ng}$ (2007) [44] & $(-)$ & GlideScope & Randomized, 106 & 52 & 52 & 0 & No data & $100 \%$ & Mean 43 & No data \\
\hline & & & GlideScope & & & & & & (95\% Cl 36.9 to 49.1$)$ & \\
\hline Xue (2007) [58] & na & GlideScope & Observational, Case & 91 & 79 & 12 & $19 / 27$ & $74 \%$ & Mean 38 & $97 \% 1$ st \\
\hline & & & Sel & & & & & Mac DL 11\% & (95\% Cl 35.7 to 40.3 ) & $100 \% \mathrm{OA}$ \\
\hline Malik (2008) [59] & $(+)$ & GlideScope & Randomized, 120 & 30 & 30 & 0 & No data & $70 \%$ & Mean 18.9 & No data \\
\hline & & & Pentax AWS v Mac DL & & & & & Mac DL 20\% & (95\% Cl 16.7 to 21.9) & \\
\hline
\end{tabular}


Table 1 Data extraction (Continued)

\begin{tabular}{|c|c|c|c|c|c|c|c|c|c|c|}
\hline & & & $\begin{array}{l}\text { v Truview, Cervical } \\
\text { spine limitation (MILS) }\end{array}$ & & & & & & & \\
\hline \multirow[t]{2}{*}{ Tremblay (2008) [60] } & \multirow[t]{2}{*}{ na } & \multirow[t]{2}{*}{ GlideScope } & \multirow{2}{*}{$\begin{array}{l}\text { Observational, Case } \\
\text { series, } 400 \text { subjects, } \\
\text { Mac DL then } \\
\text { GlideScope }\end{array}$} & \multirow[t]{2}{*}{400} & \multirow[t]{2}{*}{347} & \multirow[t]{2}{*}{53} & \multirow[t]{2}{*}{26} & $90 \%$ & Mean 21 & $84 \% 1$ st \\
\hline & & & & & & & & Mac DL 67\% & (95\% Cl 19.6 to 22.4$)$ & $99 \%$ OA \\
\hline \multirow[t]{2}{*}{ Robitaille (2008) [61] } & \multirow[t]{2}{*}{$(+)$} & \multirow[t]{2}{*}{ GlideScope } & \multirow{2}{*}{$\begin{array}{l}\text { Randomized, cross } \\
\text { over, } 20 \text { subjects, } \\
\text { cervical spine } \\
\text { limitation (MILS) }\end{array}$} & \multirow[t]{2}{*}{20} & \multirow[t]{2}{*}{ No data } & \multirow[t]{2}{*}{ No data } & \multirow[t]{2}{*}{1} & $50 \%$ & \multirow[t]{2}{*}{ No data } & \multirow[t]{2}{*}{ No data } \\
\hline & & & & & & & & Mac DL 0\% & & \\
\hline \multirow[t]{2}{*}{ Bathory (2009) [62] } & \multirow[t]{2}{*}{ na } & \multirow[t]{2}{*}{ GlideScope } & \multirow{2}{*}{$\begin{array}{l}\text { Observational, Case } \\
\text { series, } 50 \text { subjects, } \\
\text { Mac DL then } \\
\text { GlideScope, Cervical } \\
\text { spine limitation (MILS) }\end{array}$} & \multirow[t]{2}{*}{50} & \multirow[t]{2}{*}{48} & \multirow[t]{2}{*}{2} & \multirow[t]{2}{*}{50} & $8 \%$ & Median 50 & \multirow[t]{2}{*}{ No data } \\
\hline & & & & & & & & Mac DL 0\% & (IQR 41-63 s) & \\
\hline \multirow{2}{*}{$\begin{array}{l}\text { Stroumpoulis [63] } \\
\text { (2009) }\end{array}$} & \multirow[t]{2}{*}{ na } & \multirow[t]{2}{*}{ GlideScope } & \multirow{2}{*}{$\begin{array}{l}\text { Observational, Case } \\
\text { series, } 112 \text { subjects, } \\
\text { Mac DL then } \\
\text { GlideScope, }\end{array}$} & \multirow[t]{2}{*}{112} & 70 & 42 & 41 & $61 \%$ & No data & $90 \% 1$ st \\
\hline & & & & & & & & Mac DL 38\% & & $98 \%$ OA \\
\hline Lange (2009) [19] & $(+)$ & GlideScope & Randomized, 60 & 30 & 27 & 3 & 5 & $90 \%$ & Mean 17.3 & $97 \% 1$ st \\
\hline & & & Airtraq $\vee$ GlideScope & & & & & Mac DL 63\% & (95\% Cl 14.8 to 19.8$)$ & $100 \% \mathrm{OA}$ \\
\hline Liu (2009) [64] & $(+)$ & GlideScope & Randomized, 70 & 35 & 23 & 12 & 20 & $40 \%$ & Mean 71.9 & No data \\
\hline & & & $\begin{array}{l}\text { Pentax AWS), cervical } \\
\text { spine limitation (MILS) }\end{array}$ & & & & & Mac DL 20\% & (95\% Cl 55.5 to 88.3 ) & \\
\hline Maassen (2009) [65] & $(+)$ & GlideScope & & 50 & 37 & 13 & 17 & No data & Mean 33 & No data \\
\hline & & & $\begin{array}{l}\text { GlideScope } \vee \text { V-MAC v } \\
\text { McGrath, Obese }\end{array}$ & & & & & & (95\% Cl 27.9 to 38.1$)$ & \\
\hline Malik (2009) [66] & $(+)$ & GlideScope & Randomized, 75 & 25 & 0 & 25 & No data & $88 \%$ & Median 17 & $88 \% 1$ st \\
\hline & & & $\begin{array}{l}\text { subjects,Pentax AWrs v } \\
\text { GlideScope v Mac DL, } \\
\text { Risk of difficulty }\end{array}$ & & & & & & (IQR 12 to 21) & $96 \%$ OA \\
\hline Nouruzi-Sedeh (2009) & $(-)$ & GlideScope & Randomized, 200 & 100 & No data & No data & No data & $66 \%$ & Mean 63 & $93 \% 1 \mathrm{st}$ \\
\hline & & & $\begin{array}{l}\text { GlideScope, untrained } \\
\text { operators }\end{array}$ & & & & & Mac DL 32\% & (95\% Cl 57.0 to 68.9) & $100 \% \mathrm{OA}$ \\
\hline Teoh (2009) [68] & $(-)$ & GlideScope & Randomized, 140 & 70 & 62 & 8 & No data & $81 \%$ & Median 27.8 & No data \\
\hline & & & $\begin{array}{l}\text { subjects, Glidescope v } \\
\text { Pentax AWS }\end{array}$ & & & & & & (IQR 22 to 36) & \\
\hline Turkstra (2009) [69] & $(+)$ & GlideScope & Randomized, 80 & 79 & 67 & 12 & No data & $73 \%$ & Median 37 & $92 \% 1 s t$ \\
\hline & & & $\begin{array}{l}\text { alone (comparing } \\
\text { stylets) }\end{array}$ & & & & & & & $96 \%$ OA \\
\hline
\end{tabular}

$9 \%$ 1st

$7 \%$ 1st

o data

$8 \% 1 s t$

3\% 1st

o data

$2 \%$ 1st

$6 \%$ OA 
Table 1 Data extraction (Continued)

\begin{tabular}{|c|c|c|c|c|c|c|c|c|c|c|}
\hline $\begin{array}{l}\text { Van Zundert (2009) } \\
\text { [70] }\end{array}$ & $(+)$ & GlideScope & $\begin{array}{l}\text { Randomized, } 450 \\
\text { subjects, Mac DL then } \\
\text { GlideScope } \vee \text { V-MAC } \vee \\
\text { McGrath }\end{array}$ & 150 & 134 & 16 & No data & No data & $\begin{array}{l}\text { Mean } 34 \\
(95 \% \mathrm{Cl} 30.8 \text { to } 37.2)\end{array}$ & No data \\
\hline Hirabayashi (2010) [71] & $(-)$ & GlideScope & $\begin{array}{l}\text { Randomized, } 200 \\
\text { subjects, GlideScope v } \\
\text { Mac DL }\end{array}$ & 100 & No data & No data & No data & No data & $\begin{array}{l}\text { Mean } 64 \\
(95 \% \mathrm{Cl} 57.5 \text { to } 70.5)\end{array}$ & $\begin{array}{l}94 \% 1 \mathrm{st} \\
100 \% \mathrm{OA}\end{array}$ \\
\hline Serocki (2010) [72] & $(+)$ & GlideScope & $\begin{array}{l}\text { Randomized, cross- } \\
\text { over, } 120 \text { subjects } \\
\text { GlideScope } \vee \text { V-MAC v } \\
\text { Mac DL, Risk of } \\
\text { difficulty }\end{array}$ & 120 & 68 & 52 & 36 & $\begin{array}{l}36 \% \\
\text { Mac DL 0\% }\end{array}$ & $\begin{array}{l}\text { Median } 33 \\
\text { (IQR } 18 \text { to } 38 \text { ) }\end{array}$ & $\begin{array}{l}91 \% 1 \mathrm{st} \\
100 \% \mathrm{OA}\end{array}$ \\
\hline Siu (2010) [73] & na & GlideScope & $\begin{array}{l}\text { Observational, Case } \\
\text { series, } 742 \text { subjects }\end{array}$ & 742 & 408 & 256 & 78 & $62 \%$ & No data & No data \\
\hline Teoh (2010) [35] & $(+)$ & GlideScope & $\begin{array}{l}\text { Randomized, } 400 \\
\text { subjects, GlideScope v } \\
\text { Pentax AWS v CMAC } \\
\text { v Mac DL }\end{array}$ & 100 & 71 & 29 & No data & $78 \%$ & $\begin{array}{l}\text { Mean } 31 \\
\text { (95\% Cl } 28.0 \text { to } 34.0)\end{array}$ & $\begin{array}{l}91 \% 1 \mathrm{st} \\
100 \% \mathrm{OA}\end{array}$ \\
\hline Aziz (2011) [74] & na & GlideScope & $\begin{array}{l}\text { Observational, Case } \\
\text { series, } 2004 \text { subjects }\end{array}$ & 2004 & 1329 & 675 & 239 failed DL & No data & No data & No data \\
\hline Shippey (2007) [75] & na & McGrath & $\begin{array}{l}\text { Observational, Case } \\
\text { series, } 75 \text { subjects }\end{array}$ & 75 & 63 & 11 & 1 & No data & $\begin{array}{l}\text { Median } 25 \\
\text { (IQR } 18.5 \text { to 34.4) }\end{array}$ & $\begin{array}{l}93 \% 1 \mathrm{st} \\
98 \% \text { OA }\end{array}$ \\
\hline O'Leary (2008) [76] & na & McGrath & $\begin{array}{l}\text { Observational, Case } \\
\text { series, } 30 \text { subjects, } \\
\text { failed DL }\end{array}$ & 30 & No data & No data & 12 & $\begin{array}{l}77 \% \\
\text { Mac DL 3\% }\end{array}$ & No data & No data \\
\hline Maassen (2009) [65] & $(+)$ & McGrath & $\begin{array}{l}\text { Randomized, } 150 \\
\text { subjects, Mac DL then } \\
\text { GlideScope } \vee \text { V-MAC v } \\
\text { McGrath, Obese }\end{array}$ & 50 & 38 & 12 & 14 & No data & $\begin{array}{l}\text { Mean } 41 \\
(95 \% \mathrm{Cl} 33.9 \text { to } 48.1)\end{array}$ & $\begin{array}{l}8 \% 1 \mathrm{st} \\
100 \% \mathrm{OA}\end{array}$ \\
\hline $\begin{array}{l}\text { Van Zundert (2009) } \\
\text { [70] }\end{array}$ & $(+)$ & McGrath & $\begin{array}{l}\text { Randomized, } 450 \\
\text { subjects, Mac DL then } \\
\text { GlideScope } \vee \text { V-MAC v } \\
\text { McGrath }\end{array}$ & 150 & 133 & 17 & No data & No data & $\begin{array}{l}\text { Mean } 38 \\
(95 \% \mathrm{Cl} 34.3 \text { to } 41.7)\end{array}$ & $83 \% \mathrm{OA}$ \\
\hline Walker (2009) [77] & $(+)$ & McGrath & $\begin{array}{l}\text { Randomized, } 120 \\
\text { subjects, McGrath v } \\
\text { Mac DL }\end{array}$ & 60 & 58 & 2 & No data & No data & $\begin{array}{l}\text { Median } 47 \\
\text { (IQR } 39 \text { to 60) }\end{array}$ & $\begin{array}{l}95 \% \text { 1st } \\
100 \% \text { OA }\end{array}$ \\
\hline Hughes (2010) [78] & na & McGrath & $\begin{array}{l}\text { Observational, Case } \\
\text { series, } 6 \text { subjects }\end{array}$ & 6 & No data & No data & No data & No data & No data & No data \\
\hline Noppens (2010) [79] & na & McGrath & $\begin{array}{l}\text { Observational, Case } \\
\text { series, } 61 \text { subjects, } \\
\text { C\&L 3-4 failed Mac DL }\end{array}$ & 61 & No data & No data & 61 C\&L 3-4 & $\begin{array}{l}87 \% \\
\text { Mac DL 0\% }\end{array}$ & No data & $95 \%$ OA \\
\hline Asai (2008) [80] & na & $\begin{array}{l}\text { Pentax } \\
\text { AWS }\end{array}$ & $\begin{array}{l}\text { Observational, Case } \\
\text { series, } 100 \text { subjects }\end{array}$ & 100 & 100 & 0 & No data & No data & $\begin{array}{l}\text { Median } 35 \\
\text { (No IQR report) }\end{array}$ & $96 \%$ 1st, $99 \% \mathrm{OA}$ \\
\hline
\end{tabular}


Table 1 Data extraction (Continued)

\begin{tabular}{|c|c|c|c|c|c|c|c|c|c|c|}
\hline Enomoto (2008) [81] & $(+)$ & $\begin{array}{l}\text { Pentax } \\
\text { AWS }\end{array}$ & $\begin{array}{l}\text { Randomized, cross- } \\
\text { over, } 203 \text { subjects, } \\
\text { Mac DL v Pentax } \\
\text { AWS, cervical spine } \\
\text { limitation (MILS) }\end{array}$ & 203 & 194 & 9 & 22 & Mac DL 61\% & $\begin{array}{l}\text { Mean } 54 \\
(95 \% \text { Cl } 52.1 \text { to } 55.9)\end{array}$ & \\
\hline \multirow[t]{2}{*}{ Hirabayashi (2008) [82] } & \multirow[t]{2}{*}{ na } & \multirow{2}{*}{$\begin{array}{l}\text { Pentax } \\
\text { AWS }\end{array}$} & \multirow{2}{*}{$\begin{array}{l}\text { Observational, Case } \\
\text { series, } 405 \text { subjects }\end{array}$} & \multirow[t]{2}{*}{405} & \multirow[t]{2}{*}{ No data } & \multirow[t]{2}{*}{ No data } & \multirow[t]{2}{*}{16} & \multirow[t]{2}{*}{ No data } & Mean 42 & $95 \% 1 s t$ \\
\hline & & & & & & & & & (95\% Cl 3.8 to 81$)$ & $100 \% \mathrm{OA}$ \\
\hline \multirow[t]{2}{*}{ Malik (2008) [59] } & \multirow[t]{2}{*}{$(+)$} & \multirow{2}{*}{$\begin{array}{l}\text { Pentax } \\
\text { AWS }\end{array}$} & \multirow{2}{*}{$\begin{array}{l}\text { Randomized, } 120 \\
\text { subjects, Pentax AWS } \\
\vee \text { Mac } \vee \text { GS } \vee \text { Truview, } \\
\text { cervical spine } \\
\text { limitation (MILS) }\end{array}$} & \multirow[t]{2}{*}{30} & \multirow[t]{2}{*}{30} & \multirow[t]{2}{*}{0} & \multirow[t]{2}{*}{ No data } & $97 \%$ & Mean 16.7 & \multirow[t]{2}{*}{ No data } \\
\hline & & & & & & & & Mac DL 20\% & (95\% Cl 14 to 19.4$)$ & \\
\hline \multirow[t]{2}{*}{ Suzuki (2008) [83] } & \multirow[t]{2}{*}{ na } & \multirow{2}{*}{$\begin{array}{l}\text { Pentax } \\
\text { AWS }\end{array}$} & \multirow{2}{*}{$\begin{array}{l}\text { Observational, Case } \\
\text { series, } 320 \text { subjects }\end{array}$} & 320 & 265 & 55 & 46 & $99 \%$ & Mean 20.1 & $96 \% 1 s t$ \\
\hline & & & & & & & & Mac DL 55\% & (95\% Cl 19 to 21.2) & $100 \% \mathrm{OA}$ \\
\hline Asai (2009) [84] & na & Pentax & Observational, Case & 270 & 179 & 91 & 256 & $96 \%$ & No data & No data \\
\hline & & AWV & $\begin{array}{l}\text { serfes, } \\
\text { difficult Mac DLs }\end{array}$ & & & & & Mac DL 0\% & & \\
\hline Hirabayashi (2009) [85] & $(+)$ & Pentax & Randomized, 520 & 264 & No data & No data & No data & No data & Mean 44 & $96 \% 1 s t$ \\
\hline & & & $\begin{array}{l}\text { subjects, Mac DL V } \\
\text { Pentax AWS }\end{array}$ & & & & & & (95\% Cl 41.7 to 46.2$)$ & $100 \% \mathrm{OA}$ \\
\hline Liu (2009) [64] & $(+)$ & Pentax & Randomized, 70 & 35 & 25 & 10 & 19 & $97 \%$ & Mean 34.2 & $100 \% \mathrm{OA}$ \\
\hline & & AWS & $\begin{array}{l}\text { subjects, Pentax AWS } \\
\text { v GlideScope, Cervical } \\
\text { spine limitation (MILS) }\end{array}$ & & & & & Mac DL 19\% & ( $95 \% \mathrm{Cl} 25.6$ to 42.8 ) & \\
\hline Malik (2009) [48] & $(+)$ & Pentax & & 30 & 30 & 0 & No data & $100 \%$ & Median 10 & $93 \% 1 s t$ \\
\hline & & & $\begin{array}{l}\vee \text { Mac } \vee \text { CTrach, } \\
\text { cervical spine } \\
\text { limitation (MILS) }\end{array}$ & & & & & Mac DL 20\% & (IQR 8 to 15$)$ & $100 \% \mathrm{OA}$ \\
\hline Malik (2009) [66] & $(+)$ & Pentax & Randomized, 75 & 25 & 1 & 24 & No data & $100 \%$ & Median 15 & $72 \% 1 s t$ \\
\hline & & AWS & $\begin{array}{l}\text { subjects, Pentax AWS } \\
\text { v GlideScope v Mac, } \\
\text { Risk of difficulty }\end{array}$ & & & & & & (IQR 8 to 31) & $100 \% \mathrm{OA}$ \\
\hline Teoh (2009) [68] & $(+)$ & Pentax & Randomized, 140 & 70 & 60 & 10 & No data & $98 \%$ & Median 19 & $87 \% 1 s t$ \\
\hline & & & $\checkmark$ Glidescope & & & & & & (IQR 14 to 4.5 ) & $100 \mathrm{OA}$ \\
\hline Teoh (2010) [35] & $(+)$ & Pentax & Randomized, 400 & 100 & 83 & 17 & No data & $97 \%$ & Mean 20.6 & $95 \%$ 1st \\
\hline & & & $\begin{array}{l}\text { subjects, Glldescope v } \\
\text { Pentax AWS v C-MAC } \\
\vee \text { Mac DL }\end{array}$ & & & & & & (95\% Cl 18.3 to 22.9$)$ & $100 \% \mathrm{OA}$ \\
\hline Kaplan (2006) [86] & na & V-MAC & Observational, Case & 865 & No data & No data & 123 & $56 \%$ & No data & No data \\
\hline & & & Mac DL then V-MAC & & & & & Mac DL 36\% & & \\
\hline
\end{tabular}


Table 1 Data extraction (Continued)

\begin{tabular}{|c|c|c|c|c|c|c|c|c|c|c|}
\hline \multirow[t]{2}{*}{ Cavus (2009) [87] } & \multirow[t]{2}{*}{ na } & \multirow[t]{2}{*}{ C-MAC } & \multirow{2}{*}{$\begin{array}{l}\text { Observational, Case } \\
\text { series, } 60 \text { subjects }\end{array}$} & \multirow[t]{2}{*}{60} & \multirow[t]{2}{*}{42} & \multirow[t]{2}{*}{18} & \multirow[t]{2}{*}{ No data } & \multirow[t]{2}{*}{ No data } & Median 16 & $87 \% 1$ st \\
\hline & & & & & & & & & (IQR 6 to 58) & $100 \% \mathrm{OA}$ \\
\hline \multirow[t]{2}{*}{ Jungbauer (2009) [88] } & \multirow[t]{2}{*}{$(+)$} & \multirow[t]{2}{*}{ V-MAC } & \multirow{2}{*}{$\begin{array}{l}\text { Randomized, } 200 \\
\text { subjects, Mac DL v V- } \\
\text { MAC, at risk of } \\
\text { difficulty }\end{array}$} & \multirow[t]{2}{*}{100} & \multirow[t]{2}{*}{1} & \multirow[t]{2}{*}{99} & \multirow[t]{2}{*}{36} & $45 \%$ & Mean 40 & \multirow[t]{2}{*}{ No data } \\
\hline & & & & & & & & Mac DL 23\% & (95\% Cl 33.9 to 46.1$)$ & \\
\hline \multirow[t]{2}{*}{ Maassen (2009) [65] } & \multirow[t]{2}{*}{$(+)$} & \multirow[t]{2}{*}{ V-MAC } & \multirow{2}{*}{$\begin{array}{l}\text { Randomized, } 150 \\
\text { subjects, Mac DL then } \\
\text { GlideScope } \vee \text { V-MAC } \vee \\
\text { McGrath, Obese }\end{array}$} & \multirow[t]{2}{*}{50} & \multirow[t]{2}{*}{37} & \multirow[t]{2}{*}{13} & \multirow[t]{2}{*}{14} & \multirow[t]{2}{*}{ No data } & Mean 17 & \multirow[t]{2}{*}{ No data } \\
\hline & & & & & & & & & (95\% Cl 15 to 19 ) & \\
\hline \multirow{2}{*}{$\begin{array}{l}\text { Van Zundert (2009) } \\
\text { [70] }\end{array}$} & \multirow[t]{2}{*}{$(+)$} & \multirow[t]{2}{*}{ V-MAC } & \multirow{2}{*}{$\begin{array}{l}\text { Randomized, } 450 \\
\text { subjects, Mac DL then } \\
\text { GlideScope v V-MAC v } \\
\text { McGrath }\end{array}$} & \multirow[t]{2}{*}{150} & \multirow[t]{2}{*}{132} & \multirow[t]{2}{*}{18} & \multirow[t]{2}{*}{ No data } & \multirow[t]{2}{*}{ No data } & Mean 18 & \multirow[t]{2}{*}{ No data } \\
\hline & & & & & & & & & (95\% Cl 16.1 to 19.9$)$ & \\
\hline Meininger (2010) [89] & na & C-MAC & Observational, Case & 94 & No data & No data & 18 & $43 \%$ & No data & No data \\
\hline & & & Mac DL then C-MAC & & & & & Mac DL 35\% & & \\
\hline Serocki (2010) [72] & $(++)$ & V-MAC & Randomized, cross- & 120 & 68 & 52 & 36 & $31 \%$ & Median 27 & No data \\
\hline & & & $\begin{array}{l}\text { GlideScope } \vee \text { V-MAC v } \\
\text { Mac DL, Risk of } \\
\text { difficulty }\end{array}$ & & & & & Mac DL 0\% & (IQR 17 to 94) & \\
\hline
\end{tabular}


Table 2 Level of evidence summary

\begin{tabular}{|c|c|c|c|c|c|}
\hline Device & Outcome & Failed DL & $\begin{array}{l}\text { Difficult DL } \\
(C \& L>/=3)\end{array}$ & $\begin{array}{l}\text { At Higher Risk of } \\
\text { Difficult DL }\end{array}$ & Unselected \\
\hline \multirow[t]{4}{*}{ Airtraq } & Success 1 st attempt & No data & No data & $1+, 96-100 \%[23],[17],[14]$ & $1+, 93-100 \%[19],[13]$ \\
\hline & Success Overall & $3,80-100 \%$ [20], [15] & $\begin{array}{l}3,80-100 \%[20] \\
{[15]}\end{array}$ & $\begin{array}{l}1+, 96-100 \%[23],[17],[18],[14], \\
{[21],[16]}\end{array}$ & $1+, 89-100 \%[22],[19],[90],[13]$ \\
\hline & $\%$ C\&L 1 of glottis & $3,85-100 \%[20],[15]$ & $\begin{array}{l}3,89-100 \%[20] \\
{[15]}\end{array}$ & $\begin{array}{l}\text { 1+, Improvement, 90-95\% } \\
{[21],[18],[14]}\end{array}$ & $\begin{array}{l}\text { 1+, Improvement, 90-95\% } \\
{[19],[13]}\end{array}$ \\
\hline & Time to success & No data & No data & 1+, No [16],[18],[23],[21] & $1+$, No $[13],[22]$ \\
\hline \multirow[t]{6}{*}{ Bonfils } & Success 1 st attempt & $3,88 \%[27]$ & $3,88 \%[27]$ & $1-, 88 \%[29]$ & $3,89 \%[30]$ \\
\hline & & & & $3,88 \%[27]$ & \\
\hline & Success Overall & $3,96 \%[27]$ & $3,96 \%[27]$ & $1-, 82 \%[29]$ & $1-, 86-98 \%[30],[25],[28],[24]$ \\
\hline & & & & $3,96 \%[27]$ & \\
\hline & $\%$ C\&L 1 of glottis & No data & No data & 1-, Improvement, 82\% [29]) & No data \\
\hline & Time to success & No data & No data & $1-$, No $[29]$ & $1-, \mathrm{No}[28]$ \\
\hline \multirow[t]{6}{*}{ Bullard } & Success 1 st attempt & No data & $3,89 \%[32]$ & $1-, 86 \%[34]$ & $1-, 92 \%[28]$ \\
\hline & & & & $3,89 \%[32]$ & \\
\hline & Success Overall & No data & $3,98 \%[32]$ & $1-, 85-100 \%$ OA [34],[32],[33] & $1-, 92 \%$ OA [28] \\
\hline & & & & $3,98 \%[32]$ & \\
\hline & $\%$ C\&L 1 of glottis & No data & No data & No data & 1-, Improvement, 92\% [28] \\
\hline & Time to success & No data & No data & No data & $1-$, Yes $[28]$ \\
\hline \multirow[t]{4}{*}{ CTrach } & Success 1 st attempt & No data & No data & $\begin{array}{l}1-, 84-93 \%[48],[49],[36],[40], \\
{[17]}\end{array}$ & $\begin{array}{l}1-, 67-100 \%[44],[91],[39],[37], \\
{[45],[43],[46],[41]}\end{array}$ \\
\hline & Success Overall & $3,100 \%[38]$ & $3,95.8 \%[47]$ & $\begin{array}{l}1-, 90-100 \%[48],[18],[49],[42], \\
{[36],[40],[17]}\end{array}$ & $\begin{array}{l}1-, 93-100 \%[44],[91],[39],[37], \\
{[45],[43],[46],[41],[50]}\end{array}$ \\
\hline & $\%$ C\&L 1 of glottis & $3,100 \%[38]$ & $3,95.8 \%[47]$ & $\begin{array}{l}\text { 1-, Improvement, 30-98\% [41], } \\
{[48],[42],[36],[49]}\end{array}$ & $\begin{array}{l}\text { 1-, Improvement, 28-93\% [39], } \\
{[46],[41],[50],[44],[91],[45],[37]} \\
{[43]}\end{array}$ \\
\hline & Time to success & No data & No data & $1-$, No $[36],[18],[48]$ & $1-$, No $[43]$ \\
\hline \multirow[t]{4}{*}{ GlideScope } & Success 1 st attempt & No data & $3,90 \%[63]$ & $\begin{array}{l}1+16-93 \%[65],[64],[66],[54], \\
{[74],[59]}\end{array}$ & $\begin{array}{l}1-, 78-98 \%[73],[55],[56],[53], \\
{[58],[60],[44],[68],,[35],[19],[71]}\end{array}$ \\
\hline & Success Overall & $3,94 \%[74]$ & $3,98-100 \%[62,63]$ & $\begin{array}{l}1+, 89-100 \%[65],[64],[66],[54], \\
[74],[72],[59],, 661],[62]\end{array}$ & $\begin{array}{l}1-, 71-100 \%[52],[55],[56],[53], \\
{[58],[60],[92],,[67],,[51],[44],[68],} \\
{[35],[19],,[71],[93]}\end{array}$ \\
\hline & $\%$ C\&L 1 of glottis & No data & $\begin{array}{l}\text { 3, Improvement, } \\
8 \%[62]\end{array}$ & $\begin{array}{l}\text { 1+, Improvement, 33-88\% [4], } \\
[72],[64],[61],[54],[59],, 66]\end{array}$ & $\begin{array}{l}\text { 1-, Improvement, 62-100\% } \\
([73],, 67],[58],[56],[35],[53],[68], \\
{[94],[55],[60],[19],[44]}\end{array}$ \\
\hline & Time to success & No data & 3, No [62] & $1+, \mathrm{No}[59],[72]$ & $1-$, No [71] \\
\hline \multirow[t]{4}{*}{ McGrath } & Success 1 st attempt & No data & No data & No data & $1-, 93-95 \%[75],[77]$ \\
\hline & Success Overall & $\begin{array}{l}\text { 3, Improvement, 83- } \\
95 \%[76],[79]\end{array}$ & No data & No data & 1-, 98-100\% [75],[77] \\
\hline & $\%$ C\&L 1 of glottis & $\begin{array}{l}\text { 3, Improvement, 77- } \\
87 \%[76],[79]\end{array}$ & No data & No data & No data \\
\hline & Time to success & No data & No data & No data & 1-, No [77] \\
\hline \multirow[t]{4}{*}{$\begin{array}{l}\text { Pentax } \\
\text { AWS }\end{array}$} & Success 1 st attempt & $3,94 \%[84])$ & $3,94 \%[84]$ & $1+, 72-97 \%[59],[48],[95][64]$ & $\begin{array}{l}1+, 87-96 \%[80],[83][96],[85], \\
{[68],[35]}\end{array}$ \\
\hline & Success Overall & $3,99 \%[84]$ & $3,99 \%[84]$ & $\begin{array}{l}1+, 97-100 \%[59],[48],[95], \\
{[64],[81]}\end{array}$ & $\begin{array}{l}1+, 99-100 \%[80],[83],[96],[85], \\
{[68],[35]}\end{array}$ \\
\hline & $\%$ C\&L 1 of glottis & $\begin{array}{l}\text { 3, Improvement, 96\% } \\
\text { [84] }\end{array}$ & $\begin{array}{l}\text { 3, Improvement, } \\
96 \%[84]\end{array}$ & $\begin{array}{l}\text { 1+, Improvement, 97-100\% } \\
{[59],[64],[48],[81],[66]}\end{array}$ & $\begin{array}{l}\text { 1+, Improvement, 97-99\% } \\
{[68],[35],[83]}\end{array}$ \\
\hline & Time to success & No data & No data & $1+$, No $[81],[59],[95]$ & $1+$, Yes $[85],[35]$ \\
\hline V-MAC & Success 1 st attempt & No data & No data & $1+, 64 \%[65]$ & $1-, 87-93 \%[87],[68]$ \\
\hline
\end{tabular}


Table 2 Level of evidence summary (Continued)

\begin{tabular}{lllll}
\hline Success Overall & No data & No data & $1+$, 98-100\% [87],[65],[72],[88] & $1-$, 99-100\% [87],[68],[89] \\
\% C\&L $\mathbf{1}$ of glottis & No data & No data & $\begin{array}{l}1+, \text { Improvement, 31-45\% } \\
{[72],[88]}\end{array}$ & $\begin{array}{l}1-, \text { Improvement, 43-100\% } \\
{[89],[86],[35],[87]}\end{array}$ \\
& & & $1+$, No [72] & $1-$, No [35] \\
\hline
\end{tabular}

greater) to be $16 \%$ [10]. Less than 1 in 5 of those subjects with a "positive" Mallampati score 3 or 4 actually had a Cormack and Lehane view of 3 or greater when DL was performed. Another study has suggested an even lower PPV for the Mallampati score [98]. Consequently any study that solely uses the Mallampati classification to determine difficulty will have a very low incidence of true difficulty encountered at endotracheal intubation. The combination of the limited power of our current airway assessment methods to predict difficult direct laryngoscopy, with the multiple definitions of difficulty makes the subject classification a potential source of controversy. Our criterion for difficult laryngoscopy was a Cormack and Lehane view 3 and above; the definition provided by the ASA in the Practice Guidelines for Management of the Difficult Airway (2003) [99]. We accept that this is a conservative estimate of difficulty, but this criterion actually returned relatively few articles specifically examining this finding at laryngoscopy.

The level of evidence table (Table 2) was compiled by dividing the articles into four groups:

1. Unselected: this group of articles included subjects considered to have normal airways based on their airway examination and risk factors for difficult laryngoscopy. Even though this group contains a number of subjects that were proven during the study to have true difficult laryngoscopy, the outcomes were reported for the group as a whole and not specifically for this often-small subgroup preventing their specific outcomes to be analyzed.

2. At Higher risk of difficult direct laryngoscopy: these articles included only subjects suspected to have an increased likelihood of difficult intubation because of one or more airway assessment test results or the presence of obesity $(\mathrm{BMI}>35)$ or cervical spine limitation (collar or MILS). The data were reported for all those subjects fulfilling these criteria, irrespective of whether they were found to be good or poor direct laryngoscopic views during the study. As direct laryngoscopy was not performed before the videolaryngoscopy attempt the subjects had an unknown incidence of true difficult direct laryngoscopy (C\&L >/= 3).
3. Difficult direct laryngoscopy $(C \& L>/=3)$ : These articles included subjects with a documented Cormack and Lehane view III or greater on direct laryngoscopy before the intervention.

4. Failed direct laryngoscopy: These articles included subjects upon whom direct laryngoscopy failed to achieve tracheal intubation.

\section{Quality assessment of the evidence}

Before overall recommendations could be made regarding the efficacy of particular methods of VL, a measure of the quality of each study was made. This is particularly difficult regarding VL as the published studies consist of a mixture of observational (case-control and case series) with few actual randomized, controlled studies. The Agency for Healthcare Research and Quality performed a review of the many methods for assessing the quality of studies and found few that could be applied to both prospective randomized and observational studies [100]. Following their recommendations the current

Table 3 Level of evidence for overall success for devices under study

\begin{tabular}{|c|c|c|c|}
\hline & $\begin{array}{l}\text { Good } \\
\text { evidence } \\
\text { (Level 1+) }\end{array}$ & $\begin{array}{l}\text { Weak } \\
\text { evidence } \\
\text { (Level 3) }\end{array}$ & $\begin{array}{l}\text { No } \\
\text { evidence }\end{array}$ \\
\hline \multirow{5}{*}{$\begin{array}{l}\text { Subjects at higher risk of } \\
\text { difficulty during } D L\end{array}$} & Airtraq & Bonfils & \multirow[t]{5}{*}{ McGrath } \\
\hline & CTrach & Bullard & \\
\hline & GlideScope & & \\
\hline & Pentax AWS & & \\
\hline & V-MAC & & \\
\hline \multirow[t]{6}{*}{ Known difficult $D L$} & & Airtraq & McGrath \\
\hline & & Bonfils & \multirow[t]{5}{*}{ V-MAC } \\
\hline & & Bullard & \\
\hline & & CTrach & \\
\hline & & GlideScope & \\
\hline & & Pentax AWS & \\
\hline \multirow[t]{6}{*}{ Failed DL } & & Airtraq & Bullard \\
\hline & & Bonfils & \multirow[t]{5}{*}{ V-MAC } \\
\hline & & CTrach & \\
\hline & & GlideScope & \\
\hline & & McGrath & \\
\hline & & Pentax AWS & \\
\hline
\end{tabular}




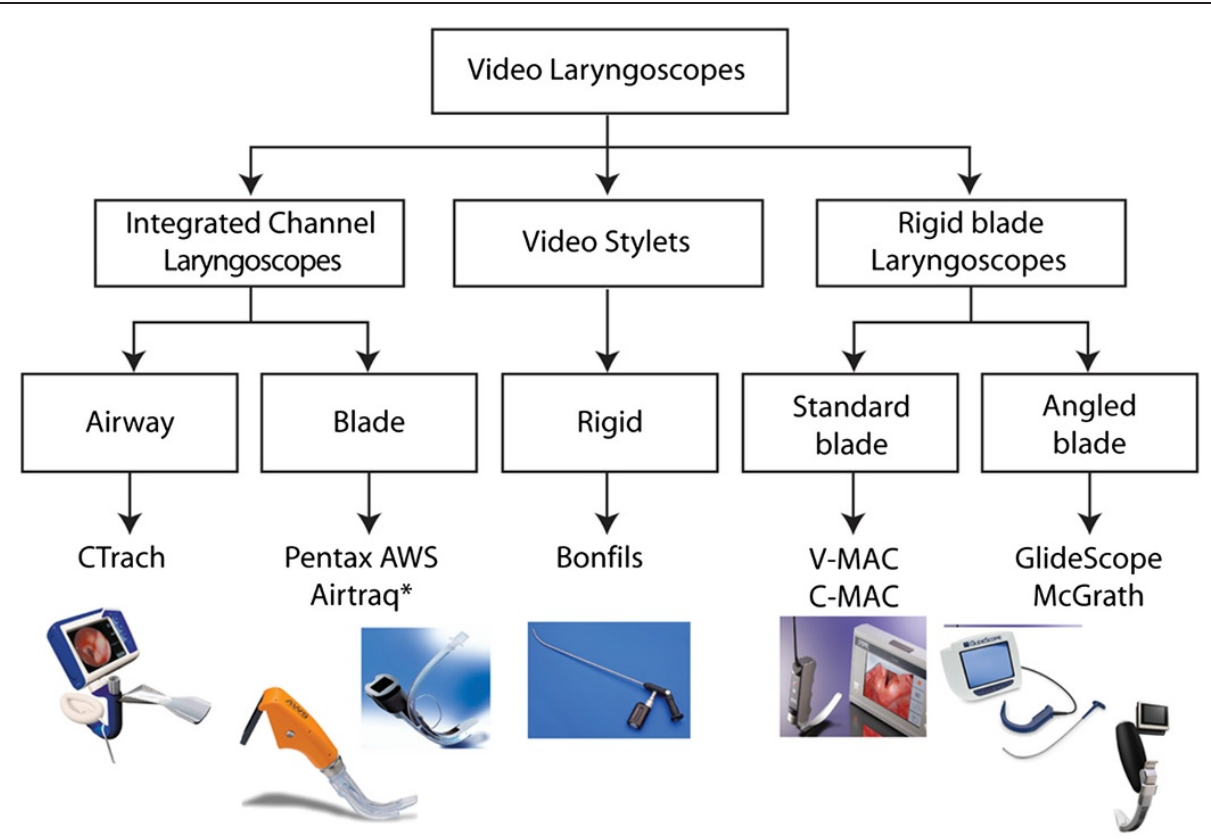

Figure 2 A classification of videolaryngoscopic devices. CTrach image courtesy of LMA North America. Pentax AWS image courtesy of Ambu USA. Airtraq image courtesy of Prodol Meditec S.A. Bonfils and C-MAC @2012 Photo Courtesy of KARL STORZ Endoscopy-America, Inc. GlideScope image courtesy of Verathon, USA. The McGrath series 5 image courtesy of Aircraft Medical, UK.

review used the methodology developed by the Scottish Intercollegiate Guidelines Network [12]. This method allowed non-analytical studies (eg. Case reports and case series) to contribute to the overall evidence (although at a much weaker score). Using defined criteria the methodological quality of each analytical study was made to give a quality rating $(++=$ good,$+=$ adequate, $-=$ poor $)$ (Table 4). Of note, the SIGN methodology does not allow a quality assessment to be made for non-analytical studies. Each study was reviewed by 2 investigators (DH, $\mathrm{OM})$ and entered into a standard data extraction table (Table 1). Where disagreement was found, this was discussed and consensus attained. Of note, a single study may appear multiple times in the data extraction table if multiple devices were investigated and data reported for each device. Evidence for one method of VL over another was presented as a level of evidence (Table 4) and then tied to a grade of recommendation in the discussion of these findings based upon the SIGN criteria [12] (Table 5).

\section{Review of the evidence}

\section{The evidence for efficacy of videolaryngoscopy}

The performance of a device when compared with direct laryngoscopy relies on three main outcomes: overall success, 1st time success, and time to successful intubation. Glottic view is a desirable outcome but intubation can remain successful and timely despite a limited view of the glottis, and in the case of VL a good laryngeal view

\section{Table 4 Levels of evidence}

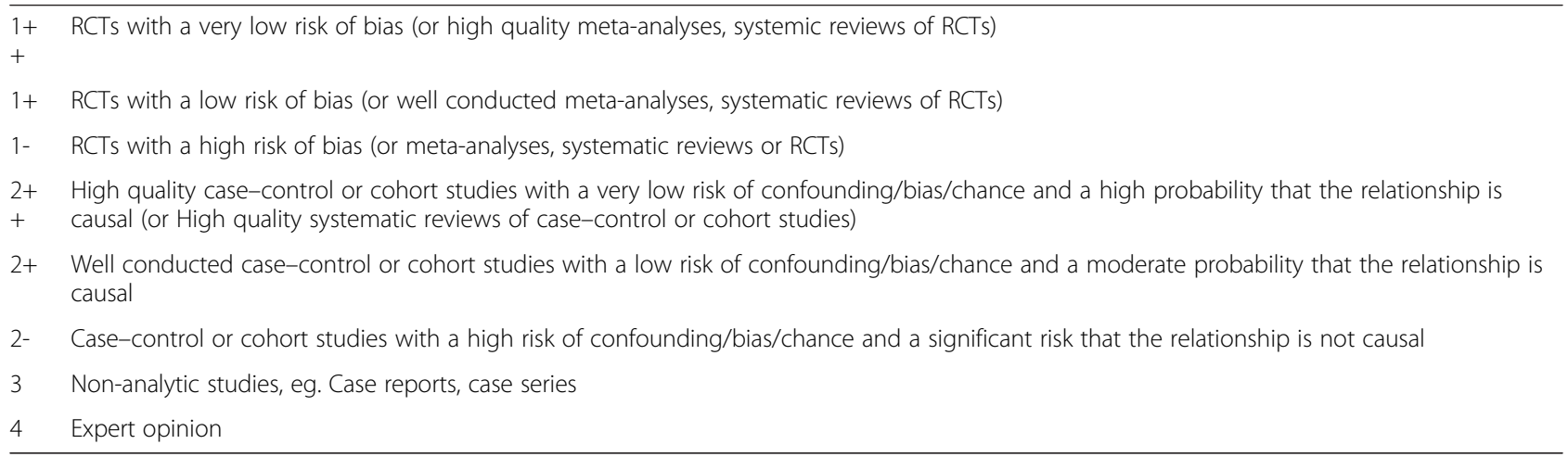

Reproduced from Harbour R, Miller J. A new system for grading recommendations in evidence based guidelines. BMJ (Clinical research ed 2001;323:334-6) with permission from BMJ Publishing Group Ltd. RCTs, Randomized controlled trials. 


\section{Table 5 Grades of recommendations}

A At least one metanalysis, systematic review, or RCT rated as 1++ and directly applicable to the target population, or A systematic review of RCTs or a body of evidence consisting principally of studies rated as $1+$ directly applicable to the target population and demonstrating overall consistency of results

B A body of evidence including studies rated as $2++$ directly applicable to the target population and demonstrating overall consistency of results, or extrapolated evidence from studies rated as $1++$ or $1+$

C A body of evidence including studies rated as $2+$ directly applicable to the target population and demonstrating overall consistency of results or extrapolated evidence from studies rated as $2++$

D Evidence level 3 or 4 , or extrapolated evidence from studies rated as $2+$

Reproduced from Harbour R, Miller J. A new system for grading recommendations in evidence based guidelines. BMJ (Clinical research ed 2001;323:334-6) with permission from BMJ Publishing Group Ltd.

does not ensure successful intubation. After careful review of the literature it was decided that little could be summarized or deduced from the Time to Intubation; this outcome being so variably defined between the studies as to make it useless as a form of comparison. We left the summary data from the outcome in the tables for completeness. Instead, we focused on overall success and first time success when compiling our evidence and recommendations, supplemented with information regarding the attainment of glottic view when possible. We followed the methodology developed by the Scottish Intercollegiate Guidelines Network [12] which was recommended by the Agency for Healthcare Research and Quality when assessing the strength of evidence provided by both prospective randomized and observational studies [100].

\section{Evidence for the use of video laryngoscopy in unselected patients}

As previously discussed, the success rate for standard direct laryngoscopy in a general, unselected population without airway pathology is likely to be greater than $95 \%$ [10]. It must be noted that there is a difference between an improvement in laryngoscopic success (ie. achieving a view of the glottis) and success of intubation. Direct laryngoscopy is often successful, despite an inadequate view of the glottis. The review of videolaryngoscopy revealed an overall success rate for unselected patients of between 94 to $100 \%$ for all of the devices, which is similar to the high success rate of direct laryngoscopy. If used to lower the incidence of difficult intubation, videolaryngoscopy may have little to offer in this unselected patient population due to the low incidence of actual difficulty encountered. However, as we will later discuss, failure can occur during any intubation attempt and the utility of video laryngoscopy must be considered as an alternative intubation device when direct laryngoscopy fails. The performance benefit of videolaryngoscopy as an educational tool was not examined in the current review, but our opinion is that the techniques of videolaryngoscopy should be practiced in a normal population, and competency demonstrated, before attempting to use in a difficult laryngoscopic scenario. There is no current evidence to suggest an increased rate of traumatic airway complications compared with direct laryngoscopy in unselected patients although there are many case reports detailing injuries and hypotheses for their causation [101-108]. The lack of evidence for a particular device should not be interpreted as evidence against its use, but rather a weakness of the published evidence.

\section{Evidence for the use of videolaryngoscopy in patients} assessed to be at high risk of difficult direct laryngoscopy When examining overall success the current review demonstrates a high rate of success when using the Airtraq, CTrach, GlideScope, Pentax AWS, and V-MAC videolaryngoscopes supported by level $1+$ evidence (good prospective). There is weaker level 3 evidence (case series) to support the use of the Bonfils and Bullard. We found no evidence for the use of the McGrath in this clinical setting. Additionally, the review revealed level $1+$ evidence (good prospective) for a higher proportion of Cormack and Lehane grade I views (compared to direct laryngoscopy) when using the Airtraq, CTrach, GlideScope, Pentax AWS, and V-MAC. The review revealed no evidence for the Bonfils, Bullard, and McGrath for the attainment of a higher proportion of $\mathrm{C} \& \mathrm{~L}$ grade I views (compared to direct laryngoscopy).

Given the above evidence, for those patients judged to be at risk of having a difficult laryngeal view on direct laryngoscopy we recommend the use of the Airtraq, CTrach, GlideScope, Pentax AWS, and V-MAC, by an operator with reasonable prior experience, to maintain overall success at intubation and increase the likelihood of Cormack and Lehane grade I views compared to direct laryngoscopy (grade A recommendation) based on the SIGN criteria [12] (Table 5). Such selection does not preclude the possibility of awake intubation in accordance with the ASA Practice Guidelines for Management of the Difficult Airway (2003)[99].

\section{Evidence for the use of videolaryngoscopy in difficult direct laryngoscopy (C\&L >/= 3)}

The current review demonstrates a high level of overall success when using the Airtraq, Bonfils, Bullard, CTrach, Glidescope, and Pentax AWS videolaryngoscopes supported by weak level 3 (case series) evidence. We found 
no evidence for success for the McGrath or V-MAC in this clinical setting. There is additional weak nonanalytic evidence (level 3) to suggest that the use of the CTrach, GlideScope, and Pentax AWS results in an increased percentage of Cormack and Lehane grade I views of the glottis. This is in broad agreement with the previous review by Mihai et al. [11].Given the above evidence, for those patients with known difficulty direct laryngoscopy (C\&L view III or IV) we cautiously recommend the use of the Airtraq, Bonfils, Bullard, CTrach, GlideScope, and Pentax AWS (grade D recommendation) by an operator with reasonable prior experience, to maintain the overall success rate of intubation based on the SIGN criteria [12]. This particular recommendation must be considered with respect to the current ASA guidelines that recommend the use of a technique which maintains spontaneous ventilation if at all possible, in patients with known or predicted difficult laryngoscopy.

\section{Evidence for the use of videolaryngoscopy as a rescue device after failed direct laryngoscopy}

After failure of initial direct laryngoscopy morbidity has been shown to increase when more than two attempts are made at laryngoscopy during emergency intubations performed beyond the operating room [109]. Perhaps, given this finding, the Difficult Airway Society of the United Kingdom suggest in their failed intubation guideline, that a provider makes no more than 2 attempts with the same device before moving on to an alternative laryngoscopic device, with the maximum number of laryngoscopic attempts limited to 4 [110]. The ASA guidelines currently do not define the maximum number of attempts with a particular device [99] but suggest that consideration be made to the use of an alternative intubation device if the primary device fails. The videolaryngoscopes would seem to fulfill the requirement of an alternative intubation device if an anesthesia provider is skilled in their use, and the device exhibits a high 1st attempt success rate. The current review demonstrates a high level of overall success, following failed intubation via direct laryngoscopy, when using the Airtraq, Bonfils, CTrach, Glidescope, McGrath, and Pentax AWS videolaryngoscopes supported by weak level 3 evidence (case series). We found no evidence for success for the Bullard or $\mathrm{V}$-MAC in this clinical setting. There is additional weak level 3 evidence (case series) for a high first attempt success rate with use of the Bonfils and Pentax AWS in this setting. There is supplemental weak nonanalytic evidence (level 3) to suggest that the use of the Airtraq, CTrach, McGrath, and Pentax AWS results in an increased percentage of Cormack and Lehane grade I views of the glottis after failed direct laryngoscopy.

Given these findings we recommend use of the Airtraq, Bonfils, CTrach, GlideScope, McGrath, and Pentax
AWS, used by an operator with reasonable prior experience, as an alternative intubation device following failed direct laryngoscopy (grade D recommendation) based on the SIGN criteria [12]. There may be extra reason to consider use of the Bonfils or Pentax AWS given their high 1st attempt success in this setting (grade D recommendation).

\section{The limitations of the current review Classification using the Mallampati as the sole predictor of difficulty during direct laryngoscopy}

The use of the Mallampati classification as the predictor of difficulty at direct laryngoscopy is an oversimplification. We have presented Shiga's work demonstrating that it is a very poor predictor of difficulty alone even when combined with other preoperative airway assessments. Unfortunately, the various predictors of difficulty at DL are variably presented in the literature, with Mallampati being the only consistently performed preoperative test. Studies examining patients with cervical spine limitation and obesity were included into the "at higher risk of difficulty group" as the authors and publishers considered these subjects to be at higher risk of difficulty.

\section{Grading the view at laryngoscopy}

It is clear the ability of a laryngoscopic device to produce a good view of the glottis is a desirable characteristic of such a device. To allow some comparison between devices we considered a Cormack and Lehane grade I view of the glottis to be beneficial irrespective of whether it is obtained by direct or indirect means. This measure allows comparison between studies as a Cormack and Lehane grade I view is reliably recorded irrespective of whether the standard Cormack and Lehane, the various forms of modified Cormack and Lehane, or the Percentage of Glottic Opening is used in its assessment. Unfortunately the other grades of laryngoscopic view (grade 2, 2a, 2b etc.) are so variably recorded as to make other comparisons impossible. The limitation of using such a strict measure of glottic view improvement is the risk missing a lesser, but perhaps clinically significant improvement in glottic view afforded by device use, for instance an improvement from a grade 3 to a $2 \mathrm{a}$ view of the glottis.

The concept of using the Cormack and Lehane classification when comparing direct laryngoscopy with the variety of methods of videolaryngoscopy is questionable. These grading schemes are designed and validated for direct laryngoscopy only; however, this measure is used throughout all of the studies, as no other alternate scheme exists. The actual difficulty in tube passage during videolaryngoscopy (unlike direct laryngoscopy) is often independent of the view obtained on the screen. Therefore, the description of the view found during videolaryngoscopy as a simple Cormack and Lehane view analogous to that found during direct laryngoscopy may be inappropriate as it doesn't 
necessarily correlate with success. We suggest that during videolaryngoscopy a grading scheme that incorporates the difficulty encountered during passage of the endotracheal tube should be used. One simple method of grading this would be to describe the difficulty (easy, difficult, or failed) with a record of the glottic view obtained (modified Cormack and Lehane) followed by the name of the device. Difficulty could be defined by the performance of multiple attempts or the use of airway adjuncts to place the tube. For example, if the procedure is difficult but ultimately successful then this could be reported as a "Difficult Grade II GlideScope; rescued with the use of a tracheal tube introducer". This information would allow decisions to be made if the use of a videolaryngoscope is contemplated at a later date, but also allow the different types of videolaryngoscopes to be more easily compared.

\section{Device variability and the comparison of efficacy}

These devices are "moving targets", i.e. new designs are continually introduced and existing designs are modified. This makes studies of older designs sometimes of questionable applicability to those currently being sold. We considered the devices in the current review to not have changed in form or function to an appreciable amount in the study period. Where new videolaryngoscope blade shapes were introduced, but failed to fulfill the inclusion criteria, they were excluded from the analysis (example the CMAC "D blade" and King Vision ${ }^{\mathrm{TM}}$ ). The differences between the devices extend toward other design features, such as the presence of a heating element to prevent fogging of the view etc. These differences cannot be easily described in a simple classification scheme.

\section{Operator performance and competency}

Experience level and competency of the operators performing laryngoscopy was not presented or accounted for during analysis in any of the studies in the review. Instead the studies generally stated that the operators were appropriately trained and experienced in the procedure. Indeed, a simple expression of an operator's number years in practice or number of previous successful intubations doesn't provide a measure of the competency of a that operator in the use of a particular device. This is a serious limitation of the studies included in this review, which limits our conclusions and applicability of our recommendations. The current review was limited to device performance in appropriately experienced users. An improvement in the success of novices with the use of these devices was beyond the scope of this review. Any recommendations for their use must be considered in this context, and in the decision-making associated with a well-considered airway management strategy.

\section{Risk of bias within studies}

When assessing the quality of randomized controlled trials particular emphasis is placed on the quality of the randomization process and blinding of subject and observer. Of these 2 factors, blinding is especially hard to address in a study design investigating videolaryngoscopy, and is generally poorly performed in the literature resulting in a universally poor score with respect to blinding. We found no article to which we could award the $1++$ level of evidence class (excellent prospective). Randomized controlled trials may not be the best method of assessing the management of rare outcomes (such as true difficult laryngoscopy or intubation) or where blinding of operator to the device under study is impossible.

\section{Risk of bias across studies}

Current methods to assess the quality of available evidence, outlined by the Agency for Healthcare Research and Quality (AHRQ) [100], are generally characterized as weighting their quality measures heavily towards randomized controlled trials. Relatively few of the methods suggested by the AHRQ actually allow observational retrospective studies to be included in any level of evidence summary. It is perhaps here where retrospective review of high quality outcome data in large databases generated by perioperative Anesthesia Information Management Systems (AIMS) can be particularly useful. Like many topics this subject likely suffers from publication bias and selective reporting within studies.

\section{Conclusion}

In conclusion, we describe a field of research limited by poor subject classification and variable outcomes. We used a reasoned scientific approach to clarify and quantify the strength of evidence to support the use of some modern videolaryngoscopic devices. We found overall limited evidence of efficacy for many of the videolaryngoscopic devices. However, our review allowed us to produce the following limited recommendations: Firstly, in patients assessed to be at higher risk of difficult laryngoscopy we recommend the use of the Airtraq, CTrach, GlideScope, Pentax AWS, and V-MAC to achieve successful intubation (Grade A recommendation [12]). Secondly, in difficult direct laryngoscopy (Cormack and Lehane view III or IV on direct laryngoscopy) we cautiously recommend the use of the Airtraq, Bonfils, Bullard, CTrach, GlideScope and Pentax AWS to achieve successful intubation (Grade D recommendation [12]) used in accordance with the ASA practice guidelines for management of the Difficult Airway. Thirdly, additional evidence exists to recommend the use of the Airtraq, Bonfils, Bullard, CTrach, Glidescope, McGrath, and Pentax AWS following failed direct laryngoscopy to achieve 
successful intubation (Grade D recommendation [12]). Additional consideration should be made to use of the Bonfils and Pentax AWS given the evidence for 1st attempt success in this setting (Grade D recommendation). Future investigation would benefit from the precise qualification of study group airway characteristics, the use of consecutive rather than unselected subjects, the measurement and standardization of operator competency, the blinding of observers, and the standardization of outcome measures. These steps would reduce bias and help interpretation and metanalysis.

Financial Support: All funding was solely from departmental sources. No external funding was solicited or used.

\section{Competing interests}

The authors have no conflicts of interest.

\section{Authors' contributions}

$\mathrm{DH}$ designed the review, extracted the data, summarized the findings, and composed the manuscript. OM extracted the data, summarized the findings, and contributed to the manuscript. DH summarized the findings. SK participated in the study design, reviewed the manuscript and coordinated the team efforts. All authors read and approved the final manuscript.

\section{Implication statement}

This systematic review of the efficacy of videolaryngoscopy in orotracheal intubation classifies the patient groups under study into four clinical entities: unselected, at higher risk of difficulty, difficult direct laryngoscopy, and failed direct laryngoscopy. The evidence of efficacy is presented and recommendations are made.

Received: 10 May 2012 Accepted: 29 November 2012

Published: 14 December 2012

\section{References}

1. Macintosh DM: A new laryngoscope. Lancet 1943, 241(6233):205.

2. Miller RA: A new laryngoscope. Anesthesiology 1941, 2(3):310-316.

3. Finucane BT, Santora AH: Principles of Airway Management. 3rd edition. Heidelberg: Springer; 2003.

4. Agro F, Barzoi G, Montecchia F: Tracheal intubation using a Macintosh laryngoscope or a GlideScope in 15 patients with cervical spine immobilization. Br J Anaesth 2003, 90(5):705-706.

5. Cormack RS, Lehane J: Difficult tracheal intubation in obstetrics. Anaesthesia 1984, 39(11):1105-1111.

6. Satava RM, Gooden SM: The impact of video endoscopy on surgical training. Am Surg 1989, 55(5):263-266.

7. Thong SY, Lim Y: Video and optic laryngoscopy assisted tracheal intubation-the new era. Anaesth Intensive Care 2009, 37(2):219-233.

8. Niforopoulou P, Pantazopoulos I, Demestiha T, Koudouna E, Xanthos T: Video-laryngoscopes in the adult airway management: a topical review of the literature. Acta Anaesthesiol Scand 2010, 54(9):1050-1061.

9. Pott $L M$, Murray WB: Review of video laryngoscopy and rigid fiberoptic laryngoscopy. Curr Opin Anaesthesiol 2008, 21(6):750-758.

10. Shiga T, Wajima Z, Inoue T, Sakamoto A: Predicting difficult intubation in apparently normal patients: a meta-analysis of bedside screening test performance. Anesthesiology 2005, 103(2):429-437.

11. Mihai R, Blair E, Kay H, Cook TM: A quantitative review and meta-analysis of performance of non-standard laryngoscopes and rigid fibreoptic intubation aids. Anaesthesia 2008, 63(7):745-760.

12. SIGN 50: A Guideline Developer's Handbook: SIGN 50: A guideline developer's handbook. In Edited by Network SIG. Edinburgh: EH7 5EA; 2008.

13. Maharaj $\mathrm{CH}, \mathrm{O}^{\prime} \mathrm{Croinin} \mathrm{D}$, Curley G, Harte BH, Laffey JG: A comparison of tracheal intubation using the Airtraq or the Macintosh laryngoscope in routine airway management: A randomised, controlled clinical trial. Anaesthesia 2006, 61(11):1093-1099.
14. Maharaj $\mathrm{CH}$, Buckley E, Harte BH, Laffey JG: Endotracheal intubation in patients with cervical spine immobilization: a comparison of Macintosh and Airtraq laryngoscopes. Anesthesiology 2007, 107(1):53-59.

15. Maharaj CH, Costello JF, McDonnell JG, Harte BH, Laffey JG: The Airtraq as a rescue airway device following failed direct laryngoscopy: a case series. Anaesthesia 2007, 62(6):598-601.

16. Ndoko SK, Slavov V, Tual L, Gilles D: Airtraq laryngoscope in tracheal intubation of anticipated difficult airway patients. Anesthesiology 2007, 107:A604.

17. Arslan Zl, Yildiz T, Baykara ZN, Solak M, Toker K: Tracheal intubation in patients with rigid collar immobilisation of the cervical spine: a comparison of Airtraq and LMA CTrach devices. Anaesthesia 2009, 64(12):1332-1336.

18. Dhonneur G, Abdi W, Ndoko SK, Amathieu R, Risk N, El Housseini L, Polliand C, Champault G, Combes X, Tual L: Video-assisted versus conventional tracheal intubation in morbidly obese patients. Obes Surg 2009, 19(8):1096-1101

19. Lange M, Frommer M, Redel A, Trautner H, Hampel J, Kranke P, Kehl F, Scholtz LU, Roewer N: Comparison of the Glidescope and Airtraq optical laryngoscopes in patients undergoing direct microlaryngoscopy. Anaesthesia 2009, 64(3):323-328.

20. Malin E, Montblanc J, Ynineb Y, Marret E, Bonnet F: Performance of the Airtraq laryngoscope after failed conventional tracheal intubation: a case series. Acta Anaesthesiol Scand 2009, 53(7):858-863.

21. Turkstra TP, Pelz DM, Jones PM: Cervical spine motion: a fluoroscopic comparison of the AirTraq Laryngoscope versus the Macintosh laryngoscope. Anesthesiology 2009, 111(1):97-101.

22. Chalkeidis O, Kotsovolis G, Kalakonas A, Filippidou M, Triantafyllou C, Vaikos D, Koutsioumpas E: A comparison between the Airtraq and Macintosh laryngoscopes for routine airway management by experienced anesthesiologists: a randomized clinical trial. Acta Anaesthesiol Taiwan 2010, 48(1):15-20.

23. Koh JC, Lee JS, Lee YW, Chang CH: Comparison of the laryngeal view during intubation using Airtraq and Macintosh laryngoscopes in patients with cervical spine immobilization and mouth opening limitation. Korean J Anesthesiol 2010, 59(5):314-318.

24. Halligan M, Charters P: A clinical evaluation of the Bonfils Intubation Fibrescope. Anaesthesia 2003, 58(11):1087-1091.

25. Wong P: Intubation times for using the Bonfils intubation fibrescope. $\mathrm{Br} J$ Anaesth 2003, 91(5):757. author reply 757-758.

26. Bein B, Worthmann F, Scholz J, Brinkmann F, Tonner PH, Steinfath $M$ Dorges $\mathrm{V}$ : A comparison of the intubating laryngeal mask airway and the Bonfils intubation fibrescope in patients with predicted difficult airways. Anaesthesia 2004, 59(7):668-674.

27. Bein B, Yan M, Tonner PH, Scholz J, Steinfath M, Dorges V: Tracheal intubation using the Bonfils intubation fibrescope after failed direct laryngoscopy. Anaesthesia 2004, 59(12):1207-1209.

28. Wahlen BM, Gercek E: Three-dimensional cervical spine movement during intubation using the Macintosh and Bullard laryngoscopes, the Bonfils fibrescope and the intubating laryngeal mask airway. Eur J Anaesthesiol 2004, 21(11):907-913.

29. Byhahn C, Nemetz S, Breitkreutz R, Zwissler B, Kaufmann M, Meininger D: Brief report: tracheal intubation using the Bonfils intubation fibrescope or direct laryngoscopy for patients with a simulated difficult airway. Can J Anaesth 2008, 55(4):232-237.

30. Corbanese $U$, Morossi M: The Bonfils intubation fibrescope: clinical evaluation and consideration of the learning curve. Eur J Anaesthesiol 2009, 26(7):622-624

31. Corso RM, Gambale G, Piraccini E, Petrini F: Emergency airway management using the Bonfils intubation fiberscope. Intern Emerg Med 2010, 5(5):447-449.

32. MacQuarrie K, Hung OR, Law JA: Tracheal intubation using Bullard laryngoscope for patients with a simulated difficult airway. Can J Anaesth 1999, 46(8):760-765.

33. Shulman GB, Connelly NR: A comparison of the Bullard laryngoscope versus the flexible fiberoptic bronchoscope during intubation in patients afforded inline stabilization. J Clin Anesth 2001, 13(3):182-185.

34. Nileshwar A, Thudamaladinne A: Comparison of intubating laryngeal mask airway and Bullard laryngoscope for oro-tracheal intubation in adult patients with simulated limitation of cervical movements. $\mathrm{Br} J$ Anaesth 2007, 99(2):292-296. 
35. Teoh WH, Saxena S, Shah MK, Sia AT: Comparison of three videolaryngoscopes: Pentax Airway Scope, C-MAC, Glidescope vs the Macintosh laryngoscope for tracheal intubation. Anaesthesia 2010, 65(11):1126-1132.

36. Dhonneur G, Ndoko SK, Yavchitz A, Foucrier A, Fessenmeyer C, Pollian C, Combes $X$, Tual L: Tracheal intubation of morbidly obese patients: LMA CTrach vs direct laryngoscopy. Br J Anaesth 2006, 97(5):742-745.

37. Goldman AJ, Wender R, Rosenblatt W, Theil D: The fiberoptic Intubating LMA-CTrach: an initial device evaluation. Anesth Analg 2006, 103(2):508.

38. Goldman AJ, Rosenblatt WH: The LMA CTrach in airway resuscitation: six case reports. Anaesthesia 2006, 61(10):975-977.

39. Liu EH, Goy RW, Chen FG: The LMA CTrach, a new laryngeal mask airway for endotracheal intubation under vision: evaluation in 100 patients. $\mathrm{Br} J$ Anaesth 2006, 96(3):396-400.

40. Timmermann A, Russo S, Natge U, Heuer J, Graf BM: [LMA CTrach: initial experiences in patients with difficult-to-manage airways]. Anaesthesist 2006, 55(5):528-534.

41. Timmermann A, Russo S, Graf BM: Evaluation of the CTrach-an intubating LMA with integrated fibreoptic system. Br J Anaesth 2006, 96(4):516-521.

42. Cattano D, Pesetti B, Di Salvo C, Giunta F: Evaluation of the LMA CTrach. Br J Anaesth 2007, 98(3):409. author reply 409.

43. Dhonneur G, Ndoko SK: Tracheal intubation with the LMA CTrach or direct laryngoscopy. Anesth Analg 2007, 104(1):227.

44. Ng SY, Ithnin E, Lim Y: Comparison of airway management during anaesthesia using the laryngeal mask airway CTrach and Glidescope. Anaesth Intensive Care 2007, 35(5):736-742.

45. Liu EH, Goy RW, Lim Y, Chen FG: Success of tracheal intubation with intubating laryngeal mask airways: a randomized trial of the LMA Fastrach and LMA CTrach. Anesthesiology 2008, 108(4):621-626.

46. Nickel EA, Timmermann A, Roessler M, Cremer S, Russo SG: Out-of-hospital airway management with the LMA CTrach-a prospective evaluation. Resuscitation 2008, 79(2):212-218.

47. Liu EH, Wender R, Goldman AJ: The LMA CTrach in patients with difficult airways. Anesthesiology 2009, 110(4):941-943.

48. Malik MA, Subramaniam R, Churasia S, Maharaj CH, Harte BH, Laffey JG: Tracheal intubation in patients with cervical spine immobilization: a comparison of the Airwayscope, LMA CTrach, and the Macintosh laryngoscopes. Br J Anaesth 2009, 102(5):654-661.

49. Ng BS, Goy RW, Bain JA, Chen FG, Liu EH: The impact of manual in-line stabilisation on ventilation and visualisation of the glottis with the LMA CTrach: a randomised crossover trial. Anaesthesia 2009, 64(8):894-898.

50. Swadia VN, Patel MG: Our preliminary experience with LMA C-Trach. Indian J Anaesth 2009, 53(3):312-317.

51. Cooper RM, Pacey JA, Bishop MJ, McCluskey SA: Early clinical experience with a new videolaryngoscope (GlideScope) in 728 patients. Can J Anaesth 2005, 52(2):191-198.

52. Doyle JD, Ramachandran M, Zura A, Ryckman JV, Abdelmalak B: The GlideScope video laryngoscope: clinical experience in 747 Cases. Anesthesiology 2005, 103:A842.

53. Hsiao WT, Lin YH, Wu HS, Chen CL: Does a new videolaryngoscope (glidescope) provide better glottic exposure? Acta Anaesthesiol Taiwan 2005, 43(3):147-151.

54. Lim Y, Yeo SW: A comparison of the GlideScope with the Macintosh laryngoscope for tracheal intubation in patients with simulated difficult airway. Anaesth Intensive Care 2005, 33(2):243-247.

55. Rai MR, Dering A, Verghese C: The Glidescope system: a clinical assessment of performance. Anaesthesia 2005, 60(1):60-64

56. Sun DA, Warriner CB, Parsons DG, Klein R, Umedaly HS, Moult M: The GlideScope video laryngoscope: randomized clinical trial in 200 patients. Br J Anaesth 2005, 94(3):381-384.

57. Turkstra TP, Craen RA, Pelz DM, Gelb AW: Cervical spine motion: a fluoroscopic comparison during intubation with lighted stylet, GlideScope, and Macintosh laryngoscope. Anesth Analg 2005, 101(3):910-915.

58. Xue FS, Zhang GH, Liu J, Li XY, Yang QY, Xu YC, Li CW: The clinical assessment of GlideScope in orotracheal intubation under general anesthesia. Minerva Anestesio/ 2007, 73(9):451-457.

59. Malik MA, Maharaj CH, Harte BH, Laffey JG: Comparison of Macintosh, Truview EVO2, GlideScope, and Airwayscope laryngoscope use in patients with cervical spine immobilization. Br J Anaesth 2008, 101(5):723-730.

60. Tremblay MH, Williams S, Robitaille A, Drolet P: Poor visualization during direct laryngoscopy and high upper lip bite test score are predictors of difficult intubation with the GlideScope videolaryngoscope. Anesth Analg 2008, 106(5):1495-1500.

61. Robitaille A, Williams SR, Tremblay MH, Guilbert F, Theriault M, Drolet P: Cervical spine motion during tracheal intubation with manual in-line stabilization: direct laryngoscopy versus GlideScope videolaryngoscopy. Anesth Analg 2008, 106(3):935-941.

62. Bathory I, Frascarolo P, Kern C, Schoettker P: Evaluation of the GlideScope for tracheal intubation in patients with cervical spine immobilisation by a semi-rigid collar. Anaesthesia 2009, 64(12):1337-1341.

63. Stroumpoulis K, Pagoulatou A, Violari M, Ikonomou I, Kalantzi N, Kastrinaki K, Xanthos T, Michaloliakou C: Videolaryngoscopy in the management of the difficult airway: a comparison with the Macintosh blade. Eur J Anaesthesiol 2009, 26(3):218-222.

64. Liu EH, Goy RW, Tan BH, Asai T: Tracheal intubation with videolaryngoscopes in patients with cervical spine immobilization: a randomized trial of the airway scope(R) and the glidescope(R). $\mathrm{Br} J$ Anaesth 2009, 103(3):446-451.

65. Maassen R, Lee R, Hermans B, Marcus M, van Zundert A: A comparison of three videolaryngoscopes: the Macintosh laryngoscope blade reduces, but does not replace, routine stylet use for intubation in morbidly obese patients. Anesth Analg 2009, 109(5):1560-1565.

66. Malik MA, Subramaniam R, Maharaj CH, Harte BH, Laffey JG: Randomized controlled trial of the Pentax AWS, Glidescope, and Macintosh laryngoscopes in predicted difficult intubation. $\mathrm{Br} J$ Anaesth 2009, 103(5):761-768.

67. Nouruzi-Sedeh P, Schumann M, Groeben H: Laryngoscopy via Macintosh blade versus GlideScope: success rate and time for endotracheal intubation in untrained medical personnel. Anesthesiology 2009, 110(1):32-37.

68. Teoh WH, Shah MK, Sia AT: Randomised comparison of Pentax AirwayScope and GlideScope for tracheal intubation in patients with normal airway anatomy. Anaesthesia 2009, 64(10):1125-1129.

69. Turkstra TP, Jones PM, Ower KM, Gros ML: The Flex-lt stylet is less effective than a malleable stylet for orotracheal intubation using the GlideScope. Anesth Analg 2009, 109(6):1856-1859.

70. van Zundert $A$, Maassen $R$, Lee $R$, Willems $R$, Timmerman M, Siemonsma M, Buise M, Wiepking M: A Macintosh laryngoscope blade for videolaryngoscopy reduces stylet use in patients with normal airways Anesth Analg 2009, 109(3):825-831.

71. Hirabayashi Y, Otsuka Y, Seo N: GlideScope videolaryngoscope reduces the incidence of erroneous esophageal intubation by novice laryngoscopists. $J$ Anesth 2010, 24(2):303-305.

72. Serocki G, Bein B, Scholz J, Dorges V: Management of the predicted difficult airway: a comparison of conventional blade laryngoscopy with video-assisted blade laryngoscopy and the GlideScope. Eur J Anaesthesio/ 2010, 27(1):24-30.

73. Siu LW, Mathieson E, Naik VN, Chandra D, Joo HS: Patient- and operatorrelated factors associated with successful GlideScope intubations: a prospective observational study in 742 patients. Anaesth Intensive Care 2010, 38(1):70-75.

74. Aziz MF, Healy D, Kheterpal S, Fu RF, Dillman D, Brambrink AM: Routine clinical practice effectiveness of the glidescope in difficult airway management: an analysis of 2,004 glidescope intubations, complications, and failures from two institutions. Anesthesiology 2011, 114(1):34-41.

75. Shippey B, Ray D, McKeown D: Case series: the McGrath videolaryngoscope-an initial clinical evaluation. Can J Anaesth 2007, 54(4):307-313.

76. O'Leary AM, Sandison MR, Myneni N, Cirilla DJ, Roberts KW, Deane GD: Preliminary evaluation of a novel videolaryngoscope, the McGrath series 5 , in the management of difficult and challenging endotracheal intubation. J Clin Anesth 2008, 20(4):320-321.

77. Walker L, Brampton W, Halai M, Hoy C, Lee E, Scott I, McLernon DJ: Randomized controlled trial of intubation with the McGrath Series 5 videolaryngoscope by inexperienced anaesthetists. Br J Anaesth 2009, 103(3):440-445. 
78. Hughes CG, Mathews L, Easdown J, Pandharipande PP: The McGrath video laryngoscope in unstable cervical spine surgery: a case series. J Clin Anesth 2010, 22(7):575-576

79. Noppens RR, Mobus S, Heid F, Schmidtmann I, Werner C, Piepho T: Evaluation of the McGrath Series 5 videolaryngoscope after failed direct laryngoscopy. Anaesthesia 2010, 65(7):716-720.

80. Asai T, Enomoto Y, Shimizu K, Shingu K, Okuda Y: The Pentax-AWS videolaryngoscope: the first experience in one hundred patients. Anesth Analg 2008, 106(1):257-259.

81. Enomoto $Y$, Asai $T$, Arai $T$, Kamishima K, Okuda Y: Pentax-AWS, a new videolaryngoscope, is more effective than the Macintosh laryngoscope for tracheal intubation in patients with restricted neck movements: a randomized comparative study. Br J Anaesth 2008, 100(4):544-548.

82. Hirabayashi $Y$, Seo N: Airway Scope: early clinical experience in 405 patients. J Anesth 2008, 22(1):81-85

83. Suzuki A, Toyama Y, Katsumi N, Kunisawa T, Sasaki R, Hirota K, Henderson J, Iwasaki $\mathrm{H}$ : The Pentax-AWS((R)) rigid indirect video laryngoscope: clinical assessment of performance in 320 cases. Anaesthesia 2008, 63(6):641-647.

84. Asai T, Liu EH, Matsumoto S, Hirabayashi Y, Seo N, Suzuki A, Toi T, Yasumoto K, Okuda Y: Use of the Pentax-AWS in 293 patients with difficult airways. Anesthesiology 2009, 110(4):898-904.

85. Hirabayashi $Y$, Seo N: Tracheal intubation by non-anesthesia residents using the Pentax-AWS airway scope and Macintosh laryngoscope. J Clin Anesth 2009, 21(4):268-271.

86. Kaplan MB, Hagberg CA, Ward DS, Brambrink A, Chhibber AK, Heidegger T, Lozada L, Ovassapian A, Parsons D, Ramsay J, et al: Comparison of direct and video-assisted views of the larynx during routine intubation. J Clin Anesth 2006, 18(5):357-362.

87. Cavus E, Kieckhaefer J, Doerges V, Moeller T, Thee C, Wagner K: The C-MAC videolaryngoscope: first experiences with a new device for videolaryngoscopy-guided intubation. Anesth Analg 2009.

88. Jungbauer A, Schumann M, Brunkhorst V, Borgers A, Groeben H: Expected difficult tracheal intubation: a prospective comparison of direct laryngoscopy and video laryngoscopy in 200 patients. Br J Anaesth 2009, 102(4):546-550.

89. Meininger D, Strouhal U, Weber CF, Fogl D, Holzer L, Zacharowski K, Byhahn C: [Direct laryngoscopy or C-MAC video laryngoscopy? Routine tracheal intubation in patients undergoing ENT surgery]. Anaesthesist 2010, 59(9):806-811.

90. Hirabayashi Y, Seo N: Airtraq optical laryngoscope: tracheal intubation by novice laryngoscopists. Emerg Med J 2009, 26(2):112-113.

91. Yildiz TS, Ozdamar D, Arslan I, Solak M, Toker K: The LMA CTrach in morbidly obese and lean patients undergoing gynecological procedures: a comparative study. J Anesth 2010, 24(6):849-853.

92. Lim HC, Goh SH: Utilization of a GlideScope videolaryngoscope for orotracheal intubations in different emergency airway management settings. Eur J Emerg Med 2009, 16(2):68-73.

93. Choi HJ, Kang HG, Lim TH, Chung HS, Cho J, Oh YM, Kim YM: Endotracheal intubation using a GlideScope video laryngoscope by emergency physicians: a multicentre analysis of 345 attempts in adult patients. Emerg Med J 2010, 27(5):380-382.

94. Cooper RM: The glidescope videolaryngoscope. Anaesthesia 2005, 60(10):1042.

95. Malik MA, O'Donoghue C, Carney J, Maharaj CH, Harte BH, Laffey JG: Comparison of the glidescope, the pentax AWS, and the Truview EVO2 with the Macintosh laryngoscope in experienced anaesthetists: a manikin study. Br J Anaesth 2009, 102(1):128-134.

96. Hirabayashi $Y$, Seo $\mathrm{N}$ : In-line head and neck position is preferable for tracheal intubation with the Airtraq laryngoscope compared to the sniffing position. J Anesth 2008, 22(2):189-190.

97. Ochroch EA, Hollander JE, Kush S, Shofer FS, Levitan RM: Assessment of laryngeal view: percentage of glottic opening score vs Cormack and Lehane grading. Can J Anaesth 1999, 46(10):987-990.

98. Cattano D, Panicucci E, Paolicchi A, Forfori F, Giunta F, Hagberg C: Risk factors assessment of the difficult airway: an italian survey of 1956 patients. Anesth Analg 2004, 99(6):1774-1779.

99. Practice guidelines for management of the difficult airway: an updated report by the American Society of Anesthesiologists task force on management of the difficult airway. Anesthesiology 2003, 98(5):1269-1277.

100. West S, King V, Carey T: systems to rate the strength of scientific evidence. evidence report/technology assessment no. 47 (prepared by the research triangle institute-university of North Carolina evidence- based practice center under contract no. 290-97-0011). In Evidence Report/Technology Assessment No 47 (Prepared by the Research Triangle Institute-University of North Carolina Evidence-based Practice Center under Contract No 290-97-0011). Edited by (US) AfHRaQ. Rockville, MD; 2002.

101. Cooper RM: Complications associated with the use of the GlideScope videolaryngoscope. Can J Anaesth 2007, 54(1):54-57.

102. Cross P, Cytryn J, Cheng KK: Perforation of the soft palate using the GlideScope videolaryngoscope. Can J Anaesth 2007, 54(7):588-589.

103. Hsu WT, Hsu SC, Lee YL, Huang JS, Chen CL: Penetrating injury of the soft palate during GlideScope intubation. Anesth Analg 2007, 104(6):1609-1610. discussion 1611.

104. Leong WL, Lim Y, Sia AT: Palatopharyngeal wall perforation during GlideScope intubation. Anaesth Intensive Care 2008, 36(6):870-874.

105. Malik AM, Frogel JK: Anterior tonsillar pillar perforation during glidescope video laryngoscopy. Anesth Analg 2007, 104(6):1610-1611. discussion 1611.

106. Uslu B, Damgaard Nielsen R, Kristensen BB: McGrath videolaryngoscope for awake tracheal intubation in a patient with severe ankylosing spondylitis. Br J Anaesth 2010, 104(1):118-119.

107. Vincent RD Jr, Wimberly MP, Brockwell RC, Magnuson JS: Soft palate perforation during orotracheal intubation facilitated by the GlideScope videolaryngoscope. J Clin Anesth 2007, 19(8):619-621.

108. Williams D, Ball DR: Palatal perforation associated with McGrath videolaryngoscope. Anaesthesia 2009, 64(10):1144-1145.

109. Mort TC: Emergency tracheal intubation: complications associated with repeated laryngoscopic attempts. Anesth Analg 2004, 99(2):607-613.

110. Henderson JJ, Popat MT, Latto IP, Pearce AC: Difficult Airway Society guidelines for management of the unanticipated difficult intubation. Anaesthesia 2004, 59(7):675-694.

doi:10.1186/1471-2253-12-32

Cite this article as: Healy et al:: A systematic review of the role of videolaryngoscopy in successful orotracheal intubation. BMC Anesthesiology 2012 12:32

\section{Submit your next manuscript to BioMed Central and take full advantage of:}

- Convenient online submission

- Thorough peer review

- No space constraints or color figure charges

- Immediate publication on acceptance

- Inclusion in PubMed, CAS, Scopus and Google Scholar

- Research which is freely available for redistribution

Submit your manuscript at www.biomedcentral.com/submit
C) Biomed Central 